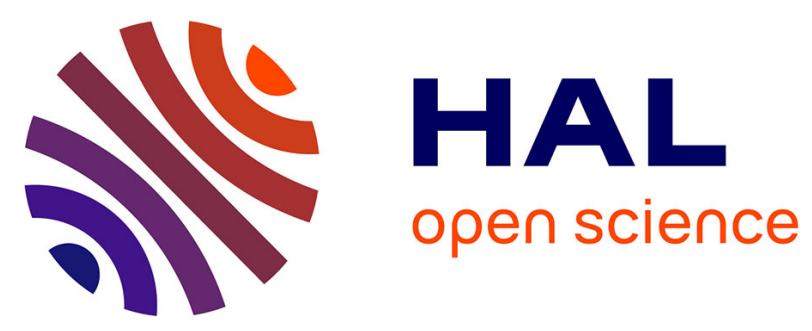

\title{
Fluid-structure interaction using a partitioned semi-implicit predictor-corrector coupling scheme for the application of large-eddy simulation
}

Michael Breuer, Guillaume de Nayer, Manuel Münsch, Thomas Gallinger, Roland Wüchner

\section{To cite this version:}

Michael Breuer, Guillaume de Nayer, Manuel Münsch, Thomas Gallinger, Roland Wüchner. Fluidstructure interaction using a partitioned semi-implicit predictor-corrector coupling scheme for the application of large-eddy simulation. Journal of Fluids and Structures, 2012, 29, pp.107-130. 10.1016/j.jfluidstructs.2011.09.003 . hal-01018330

\section{HAL Id: hal-01018330 \\ https://hal.science/hal-01018330}

Submitted on 4 Jul 2014

HAL is a multi-disciplinary open access archive for the deposit and dissemination of scientific research documents, whether they are published or not. The documents may come from teaching and research institutions in France or abroad, or from public or private research centers.
L'archive ouverte pluridisciplinaire HAL, est destinée au dépôt et à la diffusion de documents scientifiques de niveau recherche, publiés ou non, émanant des établissements d'enseignement et de recherche français ou étrangers, des laboratoires publics ou privés.

\section{(1) (1) $\$$}

Distributed under a Creative Commons Attribution - NonCommercial - NoDerivatives| 4.0 


\title{
Fluid-structure interaction using a partitioned semi-implicit predictor-corrector coupling scheme for the application of large-eddy simulation
}

\author{
M. Breuer ${ }^{\mathrm{a}, *}$, G. De Nayer ${ }^{\mathrm{a}}$, M. Münsch ${ }^{\mathrm{b}}$, T. Gallinger ${ }^{\mathrm{c}}, \mathrm{R}$. Wüchner ${ }^{\mathrm{c}}$ \\ ${ }^{a}$ Professur für Strömungsmechanik, Helmut-Schmidt-Universität Hamburg, D-22043 Hamburg, Germany \\ ${ }^{b}$ Lehrstuhl für Strömungsmechanik, Universität Erlangen-Nürnberg, D-91058 Erlangen, Germany \\ ${ }^{c}$ Lehrstuhl für Statik, Technische Universität München, D-80290 München, Germany
}

\begin{abstract}
The paper is concerned with an efficient partitioned coupling scheme developed for dynamic fluid-structure interaction problems in turbulent flows predicted by eddy-resolving schemes such as large-eddy simulation (LES). To account for the added-mass effect for high density ratios of the fluid to the structure, the semi-implicit scheme guarantees strong coupling among flow and structure, but also maintains the advantageous properties of explicit time-marching schemes often used for turbulence simulations. Thus by coupling an advanced LES code for the turbulent fluid flow with a code especially suited for the prediction of shells and membranes, an appropriate tool for the time-resolved prediction of instantaneous turbulent flows around light, thin-walled structures results. Based on an established benchmark case in laminar flow, i.e., the flow around a cylinder with an attached flexible structure at the backside, the entire methodology is analyzed thoroughly including a grid independence study. After this validation, the benchmark case is finally extended to the turbulent flow regime and predicted as a coupled FSI problem applying the newly developed scheme based on a predictor-corrector method. The entire methodology is found to be stable and robust. The turbulent flow field around the flexible structure and the deflection of the structure itself are analyzed in detail.
\end{abstract}

Keywords: Fluid-structure interaction; partitioned scheme; large-eddy simulation; FSI benchmark; (artificial) added-mass effect; semi-implicit scheme

\section{Introduction}

Fluid-structure interaction is a topic of major interest in many fields such as mechanical engineering, civil engineering or medicine technique to mention only a few. Beside experimental investigations numerical simulations have become an important and valuable tool for solving this kind of problems. Two issues have led to a a great leap forward in the last years. On the one hand the numerical algorithms for the single fields experience substantial progress. On the other hand the available computational resources have strongly increased. Both developments now allow to tackle practically relevant dynamic fluid-structure interaction problems involving turbulent flow fields.

The long-term objective of the present investigation is to tackle civil engineering FSI applications involving lightweight structures such as awnings, large umbrellas, and tent roofs, exposed

\footnotetext{
${ }^{*}$ Corresponding author

Email address: breuer@hsu-hh.de (M. Breuer)
} 
to the turbulent wind field. Right from the start it was clear that highly advanced solvers for both subtasks are required for this purpose, i.e., a specialized (finite-element) solver for shells and membranes (Bletzinger et al., 2006; Fischer et al., 2010), and a specialized (finite-volume) solver relying on eddy-resolving methods such as large-eddy simulation (LES) (Breuer, 2002). Thus monolithic coupling (see, e.g. Heil, 2004) is ruled out and a partitioned scheme is favored. This idea goes back to the early work of Felippa and Park, (e.g., Park et al., 1977; Felippa and Park, 1980; Park, 1980; Park and Felippa, 1983; Felippa and Geers, 1988) named Staggered Solution Procedure. The strength of this approach originates from its modularity and the capability of using for each sub-problem the most adapted method to its mathematical properties. Examples of successful applications of this general concept are too numerous to be reviewed here. Furthermore, much work has been dedicated to the improvement of this concept (see, e.g., Piperno et al., 1995). Partitioned schemes are further classified into loosely and strongly coupled schemes. If the fluid dynamical problem and the structural problem are solved in a staggered manner without any subiterations, the coupling conditions at the interface are not exactly satisfied at each time step. The scheme is called loosely coupled and works in particular in aeroelastic applications or in applications involving compressible viscous fluids (see, e.g., Farhat, 2004; Farhat et al., 2006). However, in many other cases weakly coupled schemes were found to be apparently unstable. Involving an incompressible viscous fluid and certain density ratios between the fluid and the structure, the so-called (artificial) added-mass effect may become an issue of major concern and strongly coupled schemes are essential (see, e.g., Causin et al., 2005; Förster et al., 2007; Förster, 2007) as in the current study. During the last years a variety of publications have been devoted to the development of efficient coupling algorithms for the non-linear FSI problem arising from the strongly coupled concept. Among these are the fixed-point iterations with fixed or dynamic underrelaxation (e.g., Mok, 2001; Küttler and Wall, 2008; Degroote et al., 2008), vector extrapolation (e.g., Küttler and Wall, 2009) or Newton-based methods. For the latter recent overviews can be found in Küttler (2009), Gallinger (2010) and Vierendeels et al. (2010).

Fernández et al. (2007) suggested just another coupling concept which they call a partially explicit or semi-implicit scheme. Since that has a lot in common with the scheme proposed independently in Breuer and Münsch $(2008,2010)$ building the basis for the present paper, a brief overview should be provided. Applying the projection method developed by Chorin (1968) and Temam $(1969 \mathrm{a}, \mathrm{b})$ for the solution of the incompressible fluid flow, this scheme is extended towards FSI problems. They proposed to strongly couple the projection sub-step carried out in a known fluid domain with the structure, while the ALE advection-diffusion sub-step is only weakly, i.e. explicitly, coupled to the structure. Hence the added-mass effect is accounted for in an implicit manner, but an implicit treatment of the ALE advection-diffusion sub-step known to be particularly expensive is avoided. Starting with the extrapolation of the fluid-structure interface (step 0), the new fluid domain is defined in step 1 followed by the explicit solution of the advection-diffusion problem (step 2). The projection step consisting of the solution of the pressure equation and the governing equation for the structure, are sub-iterated in step 3 . In a rigorous theoretical evaluation the conditional stability of the scheme was proven for a linear model problem. Furthermore, the efficiency of the method compared to fully implicitly coupled schemes was shown in different numerical experiments on laminar flows using a finite-element discretization on both fields (Fernández et al., 2007).

In the present work we are interested in the simulation of turbulent flows including FSI. Direct numerical simulation and Reynolds-Averaged Navier-Stokes (RANS) equations with statistical turbulence models are not applicable either due to their enormous CPU-time requirements or their inability to predict non-equilibrium turbulent flows. Eddy-resolving schemes such as 
large - eddy simulation (LES) or hybrid LES-RANS approaches as for example detached-eddy simulation (DES) have become popular for this purpose due to their favorable capabilities of predicting complex turbulent flows (Breuer, 2002). That is especially true for instantaneous flow processes involving large-scale flow structures such as separation, reattachment and vortex shedding. Flow phenomena of such kind are very often encountered when the flow around or through a device enforces the structures to be deformed or displaced, i.e. for fluid-structure interaction. Therefore, LES is also the preferential method in the context of FSI applications and therefore considered in the present study. However, LES is not well established in the FSI context up to now. In a recently published book on modeling and simulation for FSI (Bungartz et al., 2010) only two out of 15 contributions briefly touched the topic without going into details. At least three topics are of major concern to completely marry FSI and LES:

(i) The grid movement in ALE-FSI formulations generally means that also the filter width varies in time leading to additional commutation errors. This issue was addressed in Breuer and Münsch (2010) and Münsch and Breuer (2010).

(ii) For moving grids the question arises how the grid quality can be maintained during the instantaneous simulation. It is well-known that the demands on the grid quality regarding smoothness and orthogonality are higher for LES than for RANS. On the other hand the quality has to be assured at each time step requiring efficient and robust schemes. Beside using algebraic approaches also elliptic grid smoothing techniques (Spekreijse, 1995) have to be taken into account.

(iii) Furthermore, LES differs also with respect to the time resolution issue and thus adapted coupling schemes are favorable.

The present paper is intended to contribute especially to the last issue by proposing a FSI coupling method adjusted to the requirements of LES. The LES technique is based on spatial filtering of the instantaneous Navier-Stokes equations. The large scales are predicted directly by solving the filtered Navier-Stokes equations with a time-accurate scheme involving small time steps and modeling solely the small scales. For that purpose often predictor-corrector schemes are taken into account. Thus a methodology is suggested which relies on this specific time-marching scheme while taking into consideration that a strong coupling is needed to guarantee a stable and robust algorithm.

The paper is organized as follows. At first the numerical methodologies for both single fields are described separately in $\S 2$. In the subsequent section $(\S 3)$ the coupling scheme developed is explained in detail. Then in $\S 4$ a description of the test cases is provided explaining all details required to define the cases from the physical point of view. In $\S 5$ the specific numerical setups used in the present study are given. Finally, the results are presented in $\S 6$ split up into the validation procedure based on the laminar case and the final outcome for the turbulent case.

\section{Numerical Methodology for Each Specific Field}

\subsection{Computational Fluid Dynamics (CFD)}

\subsubsection{Governing Equations}

Within a FSI application the fluid forces acting on the structure lead to the displacement or deformation of the structure. Thus the computational domain is no longer fixed but changes in 
time, which has to be taken into account. Besides other numerical techniques, the most popular one is the so-called Arbitrary Lagrangian-Eulerian (ALE) formulation. Here the conservation equations for mass and momentum, which are to be solved based on a finite-volume scheme, are re-formulated for a temporally varying domain, i.e., control volumes (CV) with timedependent volumes $V(t)$ and surfaces $S(t)$. Hence the governing equations in ALE formulation expressing the conservation of mass and momentum read:

$$
\begin{aligned}
\frac{d}{d t} \int_{V(t)} \rho^{f} d V+\int_{S(t)} \rho^{f}\left(u_{j}-u_{g, j}\right) \cdot n_{j} d S & =0 \\
\frac{d}{d t} \int_{V(t)} \rho^{f} u_{i} d V+\int_{S(t)} \rho^{f} u_{i}\left(u_{j}-u_{g, j}\right) \cdot n_{j} d S & =-\int_{S(t)}\left(\tau_{i j}+\tau_{i j}^{S G S}\right) \cdot n_{j} d S-\int_{S(t)} p \cdot n_{i} d S
\end{aligned}
$$

These are the (filtered) Navier-Stokes equations for an incompressible fluid assuming temperature-independent fluid properties. Overbars typically used to denote filtered quantities within a large-eddy simulation are omitted here. Thus, the density of the fluid is denoted by $\rho^{f}$, the pressure by $p$ and the three Cartesian components of the velocity vector by $u_{i}$. The molecular momentum transport tensor is indicated by $\tau_{i j}$ whereas $n_{j}$ describes the unit normal vector pointing outwards. In case of LES, an additional tensor denoted $\tau_{i j}^{S G S}$ has to be taken into account in the surface integral on the right-hand side of eq. (2) describing the influence of the non-resolved subgrid-scales (SGS) on the resolved flow field (see $\S 2.1 .2$ ). Since the grid is deformable, the grid velocity with which the surface of a CV is moving is taken into account via $u_{g, j}$. Here, the volume integrals now describe local changes in a CV of variable shape and thus the additional mass and momentum fluxes due to $u_{g, j}$. Since the system of equations given by (1) and (2) is not closed anymore, the unknown grid velocity $u_{g, j}$ has to be determined. To compute this grid velocity while considering the conservation principle and avoiding the loss of mass and momentum, the so-called space conservation law (SCL) (Demirdžić and Perić, 1988, 1990) or geometric conservation law (GCL) (Lesoinne and Farhat, 1996) is applied:

$$
\frac{d}{d t} \int_{V(t)} d V-\int_{S(t)} u_{g, j} \cdot n_{j} d S=0 .
$$

This extra conservation law assures that within a change of the position or the shape of a CV no space is lost. In discretized form the SCL is expressed by the swept volumes of the corresponding cell faces. Inserting eq. (3) into (1), the mass conservation equation for a fixed grid is obtained:

$$
\int_{S(t)} u_{j} \cdot n_{j} d S=0
$$

Thus the prediction of extra grid fluxes is not necessary for the mass conservation equation in the context of moving grids. The additional grid fluxes in the momentum equation, however, have to be consistently determined by applying the SCL in its discrete form denoted discrete geometric conservation laws (DGCL) (Farhat et al., 2001). In the context of the RungeKutta time-marching scheme (see § 2.1.3) applied in the present study, a consistent numerical formulation reads

$$
\int_{S(t)}\left(\rho^{f} u_{i} u_{g, j}\right) \cdot n_{j} d S \approx \sum_{k=\{e, w, n, s, t, b\}}\left(\rho^{f} u_{i, k} \frac{\delta V_{k}^{n+1}}{\Delta t}\right),
$$


where the grid fluxes are split up into six contributions according to the six surfaces of the hexahedral CV, i.e., east, west, north, south, top and bottom. Thereby the differences between the volumes of the CV between the new (superscript $n+1$ ) and the old time step (n) can be expressed by the sum over the swept volumes $\delta V_{k}$ of all surfaces. In the three-dimensional case special care is required for the determination of the swept volumes since edges of CV surfaces can turn. Thus a segmentation into six tetrahedra possessing the same diagonal (Kordulla and Vinokur, 1983) is absolutely necessary in order to guarantee the correct prediction of the swept volumes.

\subsubsection{Subgrid-Scale Modeling}

In principle, the LES concept leads to a closure problem similar to that obtained by RANS. However, the non-resolvable small scales in a LES are much less problem-dependent than the large-scale motion so that the subgrid-scale turbulence can be represented by relatively simple models, e.g., zero-equation eddy-viscosity models. Like other eddy-viscosity models the wellknown and most often used Smagorinsky model (Smagorinsky, 1963) is based on Boussinesq's approximation which describes the stress tensor $\tau_{i j}^{S G S}$ as the product of the strain rate tensor $S_{i j}{ }^{1}$ and an eddy viscosity $\nu_{T}$,

$$
\tau_{i j}^{S G S, a}=\tau_{i j}^{S G S}-\delta_{i j} \tau_{k k}^{S G S} / 3=-2 \nu_{T} S_{i j} \quad \text { with } \quad S_{i j}=\frac{1}{2}\left(\frac{\partial u_{i}}{\partial x_{j}}+\frac{\partial u_{j}}{\partial x_{i}}\right),
$$

where $\tau_{i j}^{S G S, a}$ is the anisotropic (traceless) part of the stress tensor $\tau_{i j}^{S G S}$ and $\delta_{i j}$ is the Kronecker delta. The trace of the stress tensor is added to the pressure resulting in the new pressure $P=p+\tau_{k k}^{S G S} / 3$. The eddy viscosity $\nu_{T}$ itself is a function of the strain rate tensor $S_{i j}$ and the subgrid length $l$, as

$$
\begin{gathered}
\nu_{T}=l^{2}\left|S_{i j}\right| \quad \text { with } \quad l=C_{s} \Delta\left[1-\exp \left(\frac{-y^{+}}{A^{+}}\right)^{3}\right]^{0.5}, \\
\Delta=(\Delta x \cdot \Delta y \cdot \Delta z)^{1 / 3}, \quad y^{+}=\frac{y u_{\tau}}{\nu}, \quad u_{\tau}=\sqrt{\frac{\tau_{w}}{\rho^{f}}} \text { and } A^{+}=25 .
\end{gathered}
$$

Here, $\tau_{w}, u_{\tau}$ and $y^{+}$denote the wall shear stress, the wall shear stress velocity and the dimensionless wall distance, respectively. The filter width $\Delta$ is directly coupled to the volume of the computational cell. $C_{s}$ is the well-known Smagorinsky constant which has to be prescribed as a fixed value or can be determined as a function of time and space by the dynamic procedure originally proposed by Germano et al. (1991) and later improved by several authors, e.g. Lilly (1992). In the first case, a Van Driest damping function as given in eq. (7) is required in order to take the reduction of the subgrid length $l$ near solid walls into account. Owing to minor influences of SGS modeling at the moderate Reynolds number considered in this study, in the present investigations the fixed parameter version of the Smagorinsky model is applied using the well established standard constant $C_{s}=0.1$.

As mentioned in the introduction, the grid movement in ALE-FSI formulations leads to additional commutation errors due to the temporally varying filter width. In Breuer and Münsch (2010) and Münsch and Breuer (2010) this issue was addressed by investigating the influence of deforming grids on the quality of LES predictions. For this purpose, harmonic grid movements

\footnotetext{
${ }^{1}$ Overbars omitted as mentioned above.
} 
with specific amplitudes and periods have been forced to a turbulent plane channel flow applying the Smagorinsky model as well as the dynamic model. A variety of cases was considered. In one subset of cases, the amplitude of the internal grid deformation was varied and the period of the deformations was fixed. In a second subset, computations for different cycle periods at a constant amplitude were performed. The results were compared to LES predictions on a fixed grid. Comparing both SGS models, the Smagorinsky model showed less deviations from the reference case than the dynamic model. Furthermore, the mean values were observed to be less sensitive than the second-order moments. As long as the amplitudes and frequencies of the oscillations were not too high, the impact on the flow properties was found to be minor. Generally, hints on what kind of grid motions are tolerable within an FSI application using LES were obtained.

\subsubsection{Discretization}

\section{Spatial Discretization.}

For the present study the in-house code FASTEST-3D (Durst and Schäfer, 1996; Durst et al., 1996) is used and extended for the FSI-LES application intended. The code is based on a threedimensional finite-volume scheme which is used to discretize the governing equations (1) to (5) in ALE formulation. The discretization is done on a curvilinear, block-structured bodyfitted grid with colocated variable arrangement by applying standard schemes. A midpoint rule approximation of second-order accuracy is used for the discretization of the surface and volume integrals. Furthermore, the flow variables are linearly interpolated to the cell faces leading to a second-order accurate central scheme. In order to ensure the coupling of pressure and velocity fields on non-staggered grids, the momentum interpolation technique of Rhie and Chow (1983) is used.

\section{Temporal Discretization.}

For LES small time steps are required to resolve the turbulent flow field in time which leads to a preferred usage of explicit time-marching schemes. Here, a predictor-corrector scheme of second-order accuracy forms the kernel of the fluid solver. In the predictor step an explicit three substep low-storage Runge-Kutta scheme advances the momentum equation in time leading to a prediction of the velocities $u_{i}^{*}$. These predicted velocities do not satisfy mass conservation. Thus, in the following corrector step the mass conservation equation has to be fulfilled by solving a Poisson equation for the pressure-correction based on the incomplete LU decomposition method of Stone (1968). The corrector step is repeated until a predefined convergence criterion is reached and the final velocities and the pressure of the new time step are obtained. Hence the predictor-corrector step is also a projection method as originally proposed by Chorin (1968) and Temam (1969a,b). The procedure described was developed for fixed grids. Thus the question arises how to extend this predictor-corrector scheme to moving or deforming grids. However, inserting eq. (3) into (1) the mass conservation equation for a fixed grid is obtained. Therefore, the original pressure-correction scheme applied for the solution of the incompressible Navier-Stokes equations on fixed grids has not to be changed concerning the mass conservation equation in the context of moving grids. Solely in the momentum equation the grid fluxes have to be taken into account as described above. For achieving mass conservation the pressure-correction algorithm is repeated until a predefined convergence criterion $\varepsilon$ is reached leading to the final velocities $u_{i}$ and the corresponding pressure field $p$ of the new time step. For the present algorithm and test cases, typically 3 to 8 pressurecorrection iterations are required until the mass conservation equation is numerically satisfied, e.g. $\Delta \dot{m}<\varepsilon=\mathcal{O}\left(10^{-9}\right)$. 
FASTEST-3D is highly vectorized and additionally parallelized by domain decomposition with explicit message-passing based on MPI allowing efficient computations on vector-parallel machines and SMP clusters.

\subsubsection{Grid Adaptation}

As will be shown below, the coupling between fluid and structure takes place at the interface between both domains. From the viewpoint of the CFD prediction, forces on the structure have to be provided for the CSD calculation and even of stronger impact displacements at the boundaries of the computational domain for the fluid flow are delivered by CSD. As a consequence the task is to adapt the grid of the inner computational domain based on displacements provided solely at specific boundaries, i.e., the interface with the structure. The choice of an appropriate method is subject of the following constraints: The grid adaptation has typically to be done several times during each time step. Therefore, fast and efficient methods are of great importance. On the other hand, to maintain the grid quality, i.e., orthogonality and smoothness, and according to these properties the accuracy of the numerical prediction for time-dependent and moving grids, is a mandatory issue for FSI algorithms, especially for turbulent flows using the LES approach. For moderate deformations algebraic methods are found to be a good compromise since they are extremely fast and deliver reasonable grid point distributions. Another issue for the choice of the appropriate method is the question whether the algorithm can be fully parallelized, which is the case for algebraic methods. Thus, considering all arguments, the grid adjustment is presently performed based on a transfinite interpolation (Thompson et al., 1985). It consists of three shear transformations plus a tensor-product transformation. Beside using this algebraic approach also developments on elliptic grid smoothing based on a composite mapping (Spekreijse, 1995) consisting of a non-linear transfinite algebraic transformation and an elliptic transformation are currently under development for strong grid deformations.

\subsection{Computational Structural Dynamics (CSD)}

\subsubsection{Governing Equations}

Based on the continuum mechanics assumption the dynamic equilibrium of the structure is described by the momentum equation given in a Lagrangian frame of reference. Allowing large deformations, where geometrical non-linearities are not negligible, the following boundary value problem has to be considered (Hojjat et al., 2010):

$$
\rho^{s} \frac{\partial^{2} \mathbf{d}}{\partial t^{2}}=\nabla \cdot(\mathbf{F} \cdot \mathbf{S})+\rho^{s} \mathbf{b} .
$$

Here $\rho^{s}$ denotes the density of the structure, $\mathbf{d}$ the displacement of the material point in space, $\mathbf{F}$ the material deformation gradient, $\mathbf{S}$ the second Piola-Kirchhoff stress tensor and $\mathbf{b}$ the

specific volume forces. A constitutive relation providing a link between stress and strain is required to close eq. (8). For a St. Venant-Kirchhoff material with $\mathbf{C}$ being the constitutive tensor and $\mathbf{E}$ representing the Green-Lagrange strain, the second Piola-Kirchhoff stress tensor is given by

$$
\mathbf{S}=\mathbf{C}: \mathbf{E}, \quad \mathbf{E}=\frac{1}{2}\left(\mathbf{F}^{T} \cdot \mathbf{F}-\mathbf{I}\right)
$$

\subsubsection{Discretization}

For the solution of the governing equation (8) for the structure, i.e. the computational structural dynamics (CSD), the finite-element solver Carat++ (Fischer et al., 2010; Bletzinger et al., 
2006) developed by TU Munich especially for the prediction of shell or membrane behavior is applied. Carat++ is based on several finite-element types and advanced solution strategies for form finding and non-linear dynamic problems. For the dynamic analysis, different timeintegration schemes are available, e.g. the implicit generalized- $\alpha$ method (Chung and Hulbert, 1993). In the modeling of thin-walled structures, membrane or shell elements are applied for the discretization within the finite-element model. In the current case, the plate is modeled with a seven-parameter shell element. Furthermore, special care is given to prevent locking phenomena by applying the well-known ANS and EAS methods (Bischoff et al., 2004).

Both, shell and membrane elements reflect geometrically reduced structural models with a two-dimensional representation of the mid-surface which can describe the three-dimensional physical properties by introducing mechanical assumptions for the thickness direction. Due to this reduced model additional operations are required in the structural part to transfer information between the two-dimensional structure and the three-dimensional fluid model. Thus in the case of shells, the surface of the interface is found by moving the two-dimensional surface of the structure half of the thickness normal to the surface on both sides and the closing of the volume (Bletzinger et al., 2006). On these two moved surfaces the exchange of data (see $\S 3)$ is performed consistently with respect to the shell theory (Hojjat et al., 2010).

\section{Coupling Algorithm}

\subsection{Preliminary Considerations}

The choice of the coupling scheme suggested is motivated by the following considerations and constraints:

- Both the CSD code Carat++ and the CFD code FASTEST-3D are highly adapted to their specific field of application, providing many special features. To preserve these advantages and to realize an effective coupling algorithm, a partitioned solution approach is chosen. In comparison to the monolithic approach based on a unified numerical formulation of the whole FSI problem, the partitioned one possesses a stronger universality and allows to exchange codes for each subtask if appropriate.

- The method of choice for the prediction of instantaneous non-equilibrium flow processes involving large-scale flow structures (separation, reattachment, vortex shedding) very often encountered in FSI problems, is the LES technique. In contrast to the RANS approach which is not an appropriate choice for such kind of flows, LES typically requires small time steps to resolve the turbulent flow field in time which leads to a preferred usage of explicit time-marching schemes. Here, a predictor-corrector scheme of second-order accuracy forms the kernel of the fluid solver (see $\S 2.1 .3$ ). Thus the coupling scheme has to rely on explicit predictor-corrector schemes.

- For the partitioned coupling between the fluid solver and the structure solver in general two different methodologies can be distinguished. Within the so-called loose coupling approach the fluid and the structure subproblem are only solved once per time step leading to severe stability problems in many applications. Thus the strong coupling approach accounting for the (strong) added-mass effect (see, e.g., Causin et al., 2005; Förster et al., 2007; Förster, 2007) is favored here, where the solution of both subproblems are repeated in a staggered manner until a dynamic equilibrium is achieved. 
The task is to marry FSI and LES by considering all relevant aspects mentioned. Thus a partitioned but strong coupling scheme relying on a explicit predictor-corrector scheme is requested meeting the requirements for an efficient large-eddy simulation on the one hand and a stable coupling algorithm on the other hand.

\subsection{Coupling Scheme}

In $\S 2.1 .3$ the original predictor-corrector scheme for the fluid flow is explained. For coupled FSI computations this basic algorithm is extended as depicted in Fig. 1 and suggested in a similar manner already in Fernández et al. (2007). Based on the velocity and pressure field from the corrector step, the fluid forces resulting from the pressure and the viscous shear stresses at the interface between the fluid and the structure are computed. These forces are transferred to the CSD code Carat++ using the conservative interpolation scheme described in $\S 3.3$ and implemented in CoMA.

Using the fluid forces provided via CoMA, the finite-element code Carat ++ determines the stresses in the structure and the resulting displacements $\mathbf{d}_{\text {new }}$ of the structure. This response of the structure is transferred back to the fluid solver via CoMA applying a bilinear interpolation also given in $\S 3.3$.

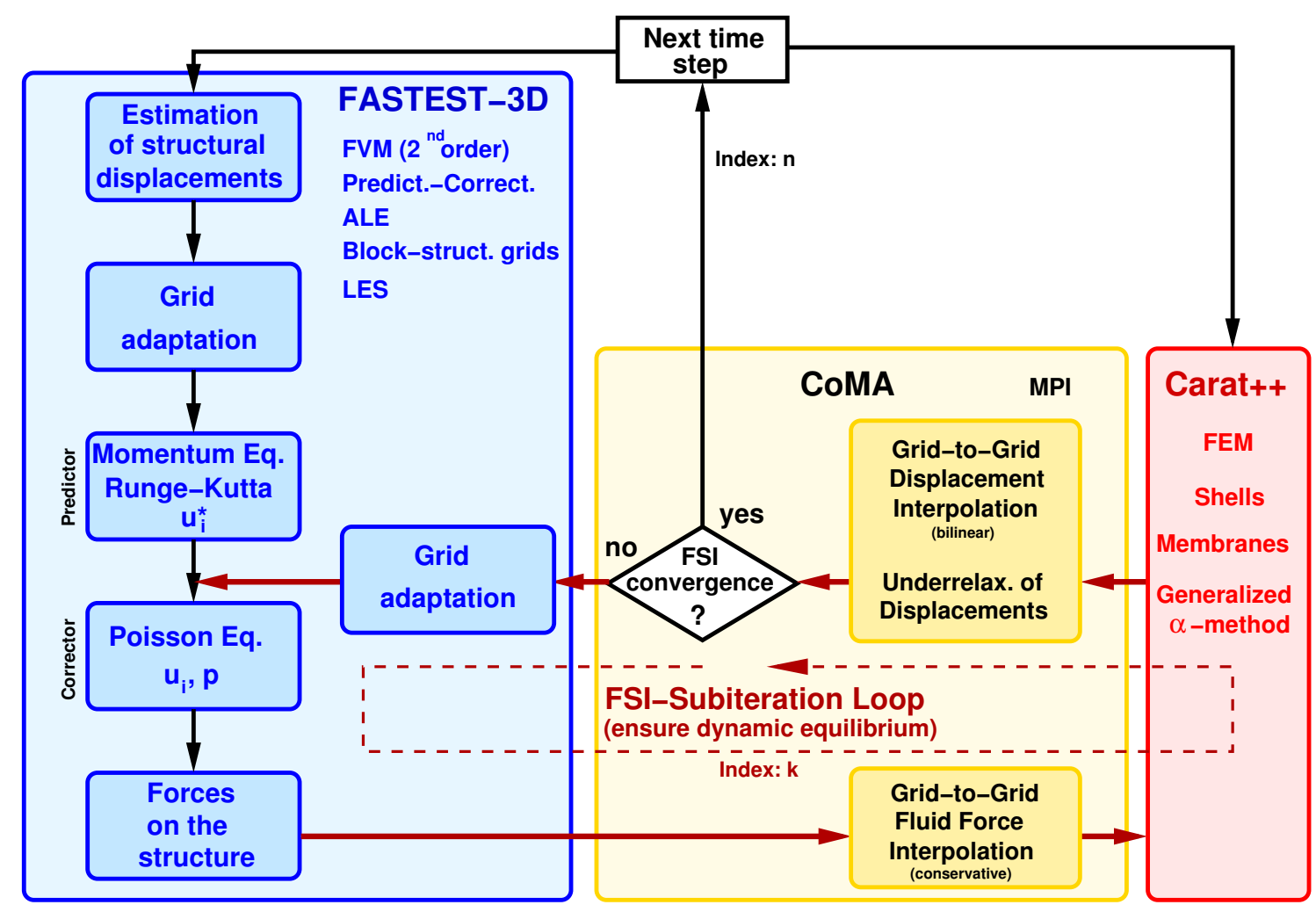

Figure 1: Sketch of the partitioned strong FSI coupling scheme including the FSI-subiteration loop.

In the case of a loose coupling approach the displacements are considered to be the physically correct displacements of the actual time step. With respect to the flowchart in Fig. 1, it means that FSI convergence is assumed to be achieved. Thus the computation would pass on to the successive time step. However, this loose coupling between fluid and structure is in general 
only stable for low density ratios of the fluid to the structure $\rho^{f} / \rho^{s} \ll 1$, i.e., in case of a low so-called added mass effect (Causin et al., 2005; Förster et al., 2007; Förster, 2007).

A typical application for this situation is encountered in aeroelasticity (Farhat, 2004), i.e., a wing of high structural density $\rho_{s}$ exposed to a fluid flow of air possessing a low fluid density $\rho_{f}$. Consequently, a low density ratio $\rho^{f} / \rho^{s}$ results. If the wing oscillates up and down, the structure has to also accelerate the fluid around the deformed or oscillating wing. That leads to additional fluid forces acting on the surface in contact with the fluid denoted as added-mass effect. Owing to the low density ratio of the air to the structure, the structure hardly senses the fluid. Thus its added mass is of minor importance and a loose coupling scheme is reasonably (Farhat, 2004; Farhat et al., 2006).

For cases of moderate and high density ratios $\rho^{f} / \rho^{s}$, e.g., a flexible structure exposed to a fluid flow of e.g. water, the structure strongly feels the surrounding fluid. Then the added-mass effect by the surrounding fluid plays a dominant role. In such situations loose coupling schemes typically tend to fail, especially in the context of incompressible flows. Thus a strong coupling scheme as suggested in the present study taking the tight interaction between the fluid and the structure into account, is indispensable. For that purpose a so-called FSI-subiteration loop is established in the coupling scheme developed (see Fig. 1) which works in the following manner:

\section{- Convergence check:}

First the FSI convergence is checked. For that purpose it has to be inspected whether the dynamic equilibrium between fluid and structure is numerically achieved. The criterion can either rely on the displacements of the structure or the loads on the interface. Presently, the change of the resulting displacements within the FSI-subiteration cycle are taken into account. Convergence is reached if the $\mathrm{L}_{2}$ norm of these displacement differences between two FSI-subiterations (index k) normalized by the $\mathrm{L}_{2}$ norm of the changes in the displacements between the current and the last time step (index n), i.e.,

$$
\frac{\left\|\mathbf{d}^{n, k}-\mathbf{d}^{n, k-1}\right\|_{2}}{\left\|\mathbf{d}^{n, k}-\mathbf{d}^{n-1}\right\|_{2}}<\varepsilon_{F S I}
$$

drops below a predefined limit, e.g. $\varepsilon_{F S I}=10^{-5}$ for the test cases of the present study.

\section{- Grid adaptation:}

Typically, convergence is not reached within the first step. Therefore, the procedure has to be continued on the fluid side. Based on the displacements on the fluid-structure interface, the inner computational CFD grid is adjusted as described in $\S$ 2.1.4.

\section{- Core of the coupling scheme / Repetition of corrector step:}

Subsequent to the grid adaptation solely the corrector step of the predictor-corrector scheme is performed again and a new velocity and pressure field is obtained. Since this issue is the key point of the coupling procedure suggested, some more explanations should be added.

In contrast to the present coupling scheme relying on explicit time marching, within a coupling scheme based on fully implicit time stepping as used, e.g., in Glück et al. (2001, 2003), an inner CFD loop becomes necessary within the applied SIMPLE scheme (Patankar and Spalding, 1972). Consequently, both schemes significantly differ with respect to the momentum equations within the FSI-subiteration loop. For the implicit scheme the momentum equations are solved repeatedly in each subiteration sweep. In contrast they are only solved once per time step for the explicit case which strongly 
reduces the computational effort. Furthermore, the number of FSI-subiterations required to reach the convergence criterion is typically much smaller for the explicit scheme (Breuer and Münsch, 2008, 2010; Münsch and Breuer, 2010) than for the implicit variant (Glück et al., 2001, 2003).

The clue is the pressure, which is the most important quantity when solving incompressible fluid flow problems. The predictor-corrector scheme guarantees that the pressure is determined in such a manner that the velocity field is finally divergence-free and mass conservation is satisfied. Furthermore, the extension of the predictor-corrector scheme towards the FSI-subiteration loops assures that the most relevant forces for the addedmass effect, i.e. the pressure forces, are successively updated until dynamic equilibrium is achieved. In conclusion, instabilities due to the added-mass effect known from loose coupling schemes are strongly reduced or completely avoided by the newly developed coupling scheme and the explicit character of the time-stepping scheme beneficial for LES is still maintained.

To concretize the comparison of both algorithms, in Glück et al. (2001) a typical number of about 1000 CFD iterations is provided for an average time step of the fully implicit scheme. That means that at each time step all three non-linear momentum equations as well as the pressure correction equation have to be solved thousand times, e.g. 4000 equations. For the present scheme, the coupling scheme is found to require only a few FSI-subiterations (5 to 10, average about 7.5) to go below the convergence limit ensuring dynamic equilibrium. As mentioned above, within each subiteration 3 to 8 (average about $5.5)$ pressure-correction iterations are necessary leading in average to $7.5 \times 5.5 \approx 41$ required solutions per time step. Together with the solution of the momentum equations a ratio of about $44 / 1000 \approx 1 / 23$ is obtained providing at least a rough estimation about the computational effort per time step required for both schemes. Thus especially for small time steps (used in LES), the scheme developed works very efficiently.

\section{- Closure of the FSI-subiteration loop:}

After a new pressure and velocity field is available, new loads for the structure solver are determined. Again these are transferred via CoMA to Carat++ leading to an update of the corresponding displacements by solving the equations of non-linear elastodynamics. The displacements are sent back via CoMA to FASTEST-3D closing the FSI-subiteration loop (see Fig. 1).

\section{- Initializing a new time step / Estimation of displacements:}

Based on the convergence criterion mentioned above, it is decided whether the subiteration process is continued or stopped. In the latter case the computation for the next time step is started. The new time step begins with an estimation of the displacement $\tilde{\mathbf{d}}$ of the structure. In contrast to the strong coupling scheme relying on a fully implicit time-marching algorithm (Glück et al., 2001, 2003), in the present scheme this measure does not only serve for convergence acceleration within the coupling process but also ensures that the momentum equations are solved on the updated geometry, which is an important feature. For the estimation two versions are taken into account. Either a firstorder linear or a second-order parabolic extrapolation is applied for the displacements by taking the displacement values of two or three former time steps indicated by the superscripts $^{n-1},{ }^{n-2}$, and ${ }^{n-3}$ into account:

$$
\begin{aligned}
\text { linear: } & \tilde{\mathbf{d}}^{n}=2 \mathbf{d}^{n-1}-1 \mathbf{d}^{n-2}, \\
\text { parabolic: } & \tilde{\mathbf{d}}^{n}=3 \mathbf{d}^{n-1}-3 \mathbf{d}^{n-2}+1 \mathbf{d}^{n-3} .
\end{aligned}
$$


Tests with the parabolic second-order extrapolation (12) have shown no improvements of the results and no better convergence than for the linear version (11). An explanation for this behavior is given by the small time-step size of the explicit time-stepping scheme.

According to these estimated boundary values, the entire computational grid has to be adapted as it is done in each FSI-subiteration loop. Once the grid is adapted, the predictor-corrector scheme of the next time step is carried out and the cycle of the FSI-subiteration loop is entered again.

Significant structural deformations can be taken into account by an underrelaxation of the boundary geometry. The grid adaptation is then based on an underrelaxation of the structural response $\mathbf{d}_{n e w}^{k}$ by taking an underrelaxation factor $\omega$ and the displacement of the previous subiteration loop $(k-1)$ into account:

$$
\mathbf{d}_{\text {new }}^{k}=\omega \mathbf{d}^{k}+(1-\omega) \mathbf{d}^{k-1},
$$

where $k$ denotes the subiteration counter. The underrelaxation factor $\omega$ can either be assumed to be constant or dynamically predicted based on the Aitken method (Aitken, 1926) adjusted for vector quantities by Irons and Tuck (1969) and Mok (2001). In the latter case the underrelaxation factor is recomputed in each iteration step based on the actual status of the coupled problem. Unfortunately, stability is not fully guaranteed and in several practical applications fixed, but suitably adjusted $\omega$ values were found to be equivalent or superior to the Aitken method (see $\S$ 6.1.4). Furthermore, it should be mentioned that in case of the underrelaxation of the displacements the fluid loads do not have to be underrelaxed during the transfer from the CFD to the CSD solver.

\subsection{Data Interpolation and Transfer}

The FSI coupling scheme requires a bilateral data exchange between CSD and CFD which is managed by the coupling interface CoMA (Gallinger et al., 2009) also developed by TU Munich. Due to different discretization techniques applied for the subtasks (finite volumes vs. finite elements) also different types of grids and different grid resolutions are used leading to non-matching surfaces meshes. Consequently, a grid-to-grid data interpolation and transfer becomes necessary. These interpolation steps include two transfers: (i) the fluid loads determined by the CFD code to the CSD code, and (ii) the structural displacements predicted by the CSD code back to the CFD code. Both are described separately.

(i) For the transfer of pressure and viscous shear forces from CFD to CSD, a conservative interpolation according to Farhat et al. (1998) is used, ensuring that the load resultants on both grids are exactly the same. The main disadvantage of this method is that in case of a coarse source grid (CFD) and very fine target grid (CSD), the loads are distributed in a non-physical way. However, in the present case this issue does not play a role since the grid used for LES (but also for the laminar case) is much finer than the CSD grid.

As mentioned in $\S 2.1 .3$ the fluid solver FASTEST-3D is based on a cell-centered variable arrangement for the pressure and the velocities, whereas the grid coordinates and displacements are cell-vertex bound. Therefore, in an initial step, the fluid loads $\mathbf{F}_{\mathbf{C C}, \mathbf{C F D}}$ predicted based on the pressure and viscous shear forces at the centers of the cells denoted CC are conservatively interpolated to the face vertices, i.e., the grid nodes (N). Note that at the CFD-CSD interface the cell centers (CC) are located in the center of the cell faces and thus in the same plane as the face vertices $(\mathrm{N})$, see Fig. 2. Thus a two-dimensional interpolation has to be carried out. That step is necessary since the data transfer in CoMA is restricted to one topology on each 
side and since the displacements are required at $\mathrm{N}$, also the loads have to be defined at these locations. The whole interpolation procedure for the fluid loads is done in two steps:

1. Conservative interpolation of the fluid loads located in the cell centers $(\mathrm{CC})$ to the cell nodes $(\mathrm{N})$ of the fluid domain $(\mathrm{CFD} \rightarrow \mathrm{CFD}$, done in FASTEST-3D, see Fig. 2):

$$
\sum_{\text {CFD nodes }} \mathbf{F}_{\mathbf{N}, \mathbf{C F D}}=\sum_{\text {cell centers }} \mathbf{F}_{\mathbf{C C}, \mathbf{C F D}}
$$

Since the cell centers (CC) are exactly located in the geometrical centers of the cell, it means that for an internal node not located at an edge the fluid load is equally distributed to its four neighboring cell nodes (N), i.e., a quarter to each node. At edges of the interface, this rule has to be adjusted accordingly.

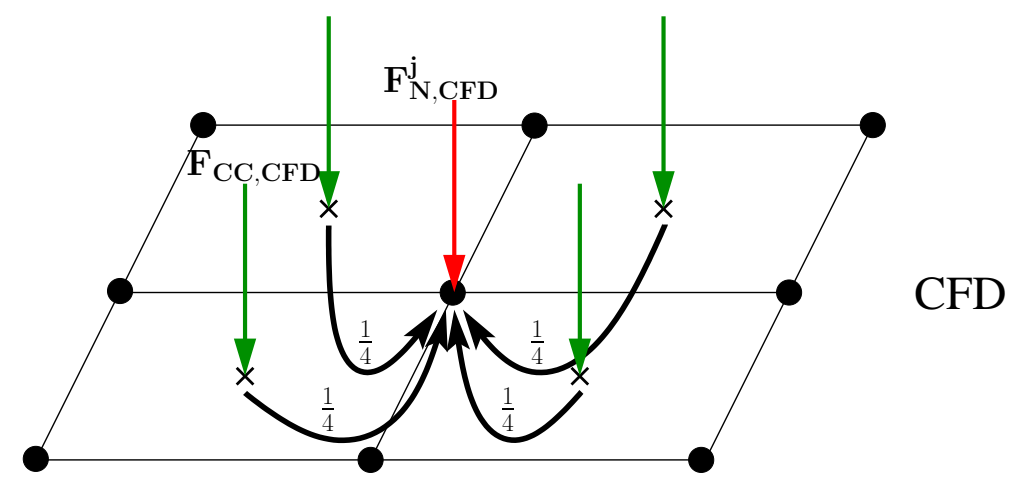

Figure 2: Interpolation of loads from the cell centers (CC) located at the CFD-CSD interface to the nodes $(\mathrm{N})$ of the CFD grid. Note: Both, $\mathrm{CC}$ and $\mathrm{N}$ lie in the same plane.

2. Conservative interpolation of the fluid loads from the cell nodes of the fluid domain to the grid nodes of the structure domain $(\mathrm{CFD} \rightarrow \mathrm{CSD}$, done in CoMA, see Fig. 3):

$$
\sum_{\text {CSD nodes }} \mathbf{F}_{\mathrm{N}, \mathrm{CSD}}=\sum_{\mathrm{CFD} \text { nodes }} \mathbf{F}_{\mathrm{N}, \mathrm{CFD}}
$$

To achieve this, the fluid load $\mathbf{F}_{\mathbf{N}, \mathbf{C F D}}^{\mathbf{j}}$ at the grid node $j$ is split up to parts which are distributed to all nodes of the CSD element $e$, in which the fluid node $j$ is found. The weighting is based on the shape function $N_{r}^{e}$ of the CSD element $e$. It reads:

$$
\mathbf{F}_{\mathbf{N}, \mathbf{C S D}}^{\mathbf{r}}=N_{r}^{e}\left(\xi_{j}, \eta_{j}\right) \mathbf{F}_{\mathbf{N}, \mathbf{C F D}}^{\mathbf{j}}
$$

(ii) The calculated displacement vectors of the CSD nodes are transferred to the CFD nodes by using a bilinear interpolation. This interpolation is a consistent scheme for four-node elements with bilinear shape functions. A conservative interpolation as used to transfer pressure and viscous shear forces is not suitable here, because the displacements are not integral quantities. Thus the final step of the interpolation procedure reads:

3. Bilinear interpolation of the displacements from the structure domain $\mathbf{d}_{\mathbf{N}, \mathbf{C S D}}^{\mathrm{r}}$ to the cell vertices of the fluid domain ( $\mathrm{CSD} \rightarrow \mathrm{CFD}$, done in CoMA, see Fig. 4):

$$
\mathbf{d}_{\mathbf{N}, \mathbf{C F D}}^{\mathbf{j}}=\sum_{r=1}^{4} N_{r}^{e}\left(\xi_{j}, \eta_{j}\right) \mathbf{d}_{\mathbf{N}, \mathbf{C S D}}^{\mathbf{r}}
$$




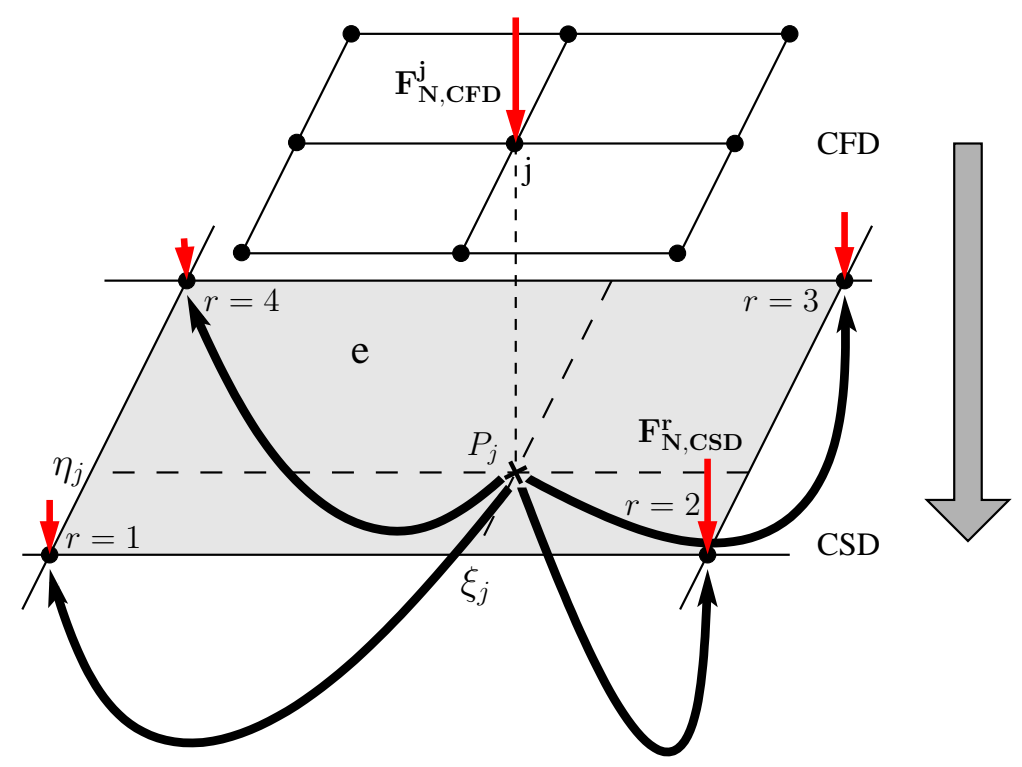

Figure 3: Transfer of loads from the fluid domain (CFD) to the structure domain (CSD).

Here, the resulting displacements in the fluid domain are denoted by $\mathbf{d}_{\mathbf{N}, \mathbf{C F D}}^{\mathbf{j}}$. They are obtained by the displacements of the structure domain $\mathbf{d}_{\mathbf{N}, \mathbf{C S D}}^{\mathbf{r}}$ weighted by the shape functions $N_{r}^{e}$ of the CSD element $e$, in which the fluid node $j$ is found. Thereby $\xi_{j}$ and $\eta_{j}$ describe the local coordinates of the projection point $P_{j}$ in the CSD element $e$.

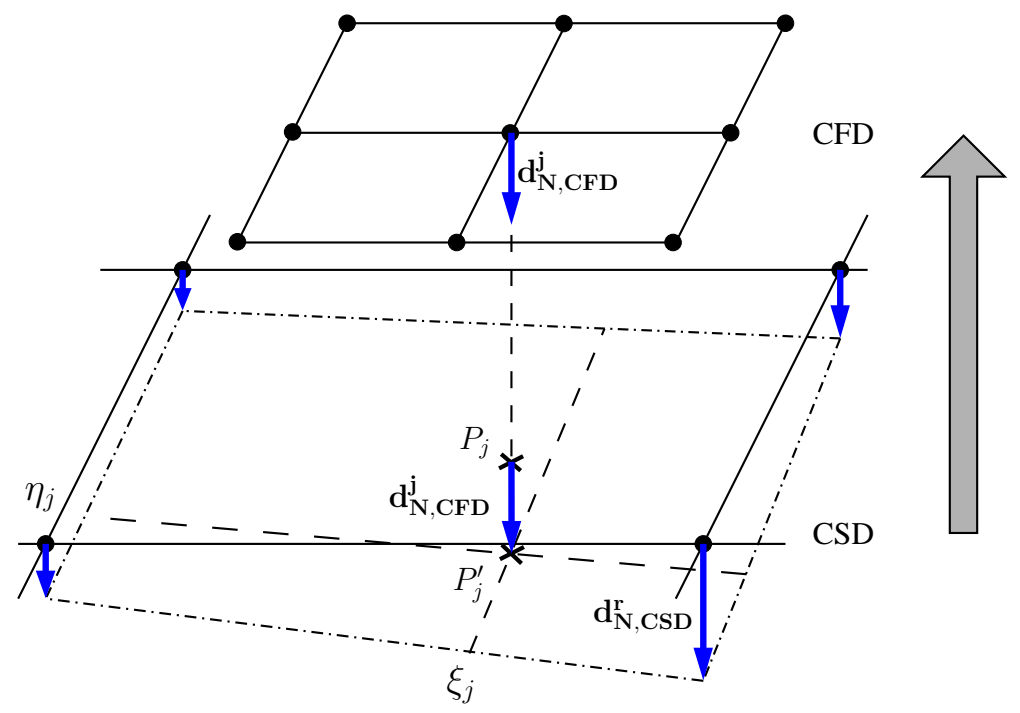

Figure 4: Transfer of displacements from the structure domain (CSD) to the fluid domain (CFD).

The interpolation steps 2 and 3 are done in CoMA. This code coupling tool is based on the Message-Passing-Interface (MPI) and thus runs in parallel to the fluid and structure solver. The communication in-between the codes is performed via standard MPI commands using a special communicator for this purpose. Since the code parallelization in FASTEST3D and Carat++ also rely on MPI, separate communicators are introduced for each code. Thus a hierarchical parallelization strategy with different levels of parallelism is achieved. 
According to the CPU time requirements of the different subtasks, an appropriate number of processors can be assigned to the fluid and the structure part. Owing to the reduced structural models on the one side and the fully three-dimensional highly resolved fluid prediction on the other side, the predominant portion of the CPU time is presently required for the CFD part. Hence in addition to the domain decomposition on the fluid and structure side, discipline decomposition is accomplished allowing the usage of modern multi-core architectures with their specific hierarchy of parallelism. Thus effective coupling with respect to high-performance computing is enabled.

\section{Test Cases}

A collaborative effort of the DFG Research Unit 493 (Bungartz et al., 2010) was the development of specific benchmark problems for validating various solution methods for fluid-structure interaction. In order to allow the application of a wide range of different coupling strategies and numerical schemes, the configuration considered is geometrically not very challenging and furthermore restricted to the laminar flow regime (Turek and Hron, 2006). One of these benchmark cases, namely FSI3, is used in this study to validate the numerical methodology proposed. The setup is described in $\S 4.1$. To go beyond the limit of laminar flows and to test the FSI scheme in the turbulent regime applying LES, the setup of FSI3 was slightly adapted. The new configuration called FSI-LES is given in $\S 4.2$

\subsection{FSI Benchmark for Laminar Flow}

The present FSI3 configuration (Turek and Hron, 2006; Turek et al., 2010) leans on an older 'flow around cylinder' benchmark developed for testing CFD methods in the laminar flow regime (Turek and Schäfer, 1996). In order to extend the original CFD benchmark to a FSI test case, a flexible structure is attached to the back side of the cylinder (Turek and Hron, 2006). In a two-dimensional channel of length $L / D=25$ and height $H / D=4.1$ a fixed and rigid cylinder of diameter $D$ is mounted as sketched in Fig. 5. The cylinder position is slightly off-centered, with the cylinder center located at $2 D$ downstream of the inflow section and with a distance of $H_{c}=2 D$ from the lower lateral channel wall. Providing dimensions the cylinder has a diameter of $D=0.1 \mathrm{~m}$. The fluid is assumed to be an incompressible Newtonian fluid with $\rho^{f}=1000 \mathrm{~kg} / \mathrm{m}^{3}$. Based on a mean inflow velocity of $u_{\infty}=2 \mathrm{~m} / \mathrm{s}$ and a kinematic viscosity of $\nu^{f}=0.001 \mathrm{~m}^{2} / \mathrm{s}$ a Reynolds number of $R e=200$ is obtained. According to the analytical solution for a fully developed laminar channel flow, a parabolic velocity profile was set at the inflow given by

$$
u(y)=1.5 u_{\infty} \frac{y(H-y)}{\left(\frac{H}{2}\right)^{2}} .
$$

Besides the parabolic inflow condition (18) for the flow, no-slip boundary conditions should be applied at the channel walls, at the rigid front cylinder and at the flexible structure (Turek and Hron, 2006). At the outlet the conditions are not prescribed in Turek and Hron (2006). Generally, a convective outflow boundary condition should be favored allowing vortices to leave the integration domain without significant disturbances (Breuer, 2002).

The elastic structure has a length of $l / D=3.5$ and a thickness of $h / D=0.2$. The structure is allowed to be compressible, and its deformations are significant. The material of the flexible structure is specified by the St. Venant-Kirchhoff material law (Belytschko et al., 2000) characterized by a Poisson's ratio of $\nu^{s}=0.4$, a Young's modulus of $E=5.6 \cdot 10^{6} \mathrm{~kg} /\left(\mathrm{m} \mathrm{s}^{2}\right)$ and a density of $\rho^{s}=1000 \mathrm{~kg} / \mathrm{m}^{3}$. Consequently, the density ratio $\rho^{f} / \rho^{s}$ is unity and the added-mass effect plays an important role. All nodes of the structure on the rigid cylinder are 
assumed to be fixed. Contrarily, the nodes of the flexible structure are totally free at the free extremity.

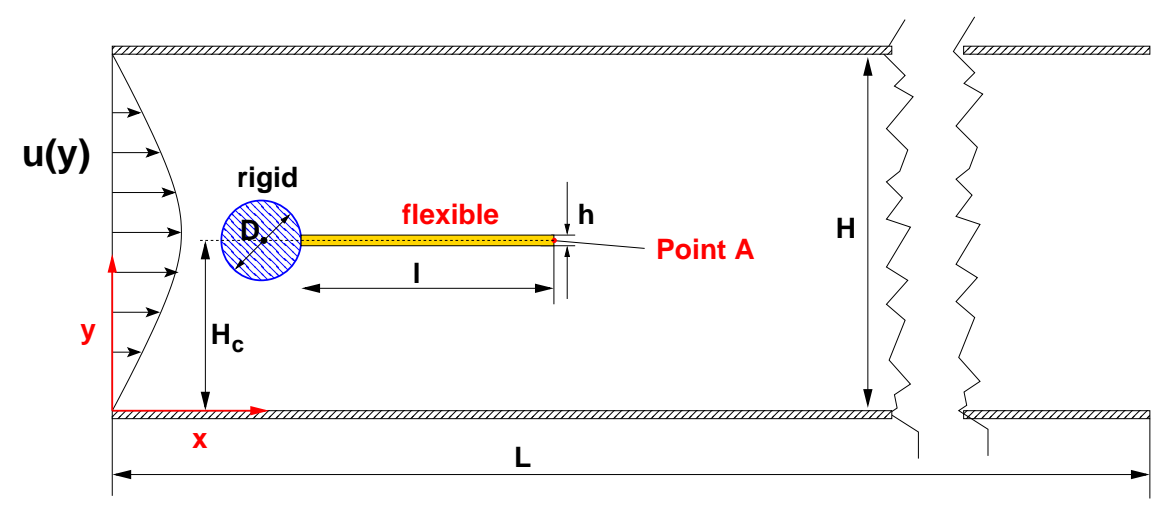

Figure 5: Setup of the computational domain for the FSI3 test case.

As a first step of a thoroughly carried out validation procedure, the single components (CFD and CSD solver) have to be addressed independently. For this issue, Turek and Hron (2006) defined the test cases CFD3 and CSM1 to CSM3, respectively. Both are briefly sketched and the results achieved will be shwon in $\S 6.1 .1$ and $\S 6.1 .2$.

\section{Benchmark CFD3.}

Assuming that the flexible structure in Fig. 5 is rigid, the FSI3 benchmark reduces to a pure CFD test case denoted CFD3 (Turek and Hron, 2006). The remainder of the test case and setup is kept unchanged. Thus the laminar two-dimensional flow around a cylinder with a kind of splitter plate at the backside mounted in a channel is considered for validation of the CFD code.

\section{Benchmarks CSM1 to CSM3.}

Similar to the validation of the CFD tool also the CSD tool is first validated in an uncoupled environment. For that purpose the benchmarks CSM1 to CSM3 (Turek and Hron, 2006) are taken into account. Based on the setup sketched in Fig. 5 solely the elastic beam without the surrounding fluid is considered here. In order to predict a deflection of the structure, a gravitational acceleration of $\mathrm{g}=2 \mathrm{~m} / \mathrm{s}^{2}$ in negative $\mathrm{y}$-direction is added. The CSM3 test is computed as a time-dependent case starting from the undeformed configuration while the tests CSM1 and CSM2 are the steady-state solutions. The material parameters are equal to those defined for FSI3 in $\S 4.1$ except the Young's modulus which is reduced to $E=1.4 \cdot 10^{6} \mathrm{~kg} /(\mathrm{m}$ $\mathrm{s}^{2}$ ) for the cases CSM1 and CSM3.

\subsection{FSI Benchmark for Turbulent Flow}

For extending the coupled FSI3 case towards an appropriate test case for the turbulent flow regime (FSI-LES), several adjustments are necessary. These are required either to allow LES or to reduce the computational effort to a reasonable level.

First, the computational domain has to be extended to a three-dimensional geometry since turbulence is always 3-D and LES predictions can thus not rely on two-dimensional integration domains. Therefore, the computational domain has now a width of $W / D=l / D=3.5$ in $\mathrm{z}^{-}$ direction. Thus the flexible structure is a square in the $\mathrm{x}-\mathrm{z}-$ plane.

Second, owing to this three-dimensional extension of the domain, boundary conditions are required for the flow and for the structure. Thus, in spanwise direction periodic boundary 
conditions are chosen for both disciplines. For the structure that implies that in the crosssection ( $\mathrm{x}-\mathrm{y}$ plane) the same boundary conditions are used as in 4.1. Additionally, the $\mathrm{z}^{-}$ displacement of the nodes on the sides are forced to be zero. Due to periodic boundary conditions set in the fluid solver there are always two nodes of the sides (one in a plane and its twin in the other plane) which have the same load. These two nodes must have the same displacement in $\mathrm{x}-$ and $\mathrm{y}$-direction.

Third, since the resolution of the boundary layers at the channel walls would require the bulk of the CPU time, the lateral channel walls are assumed to be slip walls. Thus the blocking effect of the walls is maintained without taking the boundary layers into account. Of course, on the structure, no-slip boundary conditions are used as in the laminar case. At the channel outlet again a convective outflow condition is specified to avoid reflections and disturbances (Breuer, 2002).

Fourth, as a direct consequence of the slip conditions at the channel walls, the inflow profile has to be modified and a constant velocity $u_{\infty}$ is now set at the inflow.

Fifth, in order to enter the turbulent regime, the Reynolds number is set to $R e=u_{\infty} D / \nu^{f}=10^{4}$. For the pure cylinder the flow is in the sub-critical regime at this Reynolds number, where the boundary layer at the cylinder is still laminar and transition to turbulence takes place in the free shear layers behind the cylinder (Breuer, 1998, 2000). All other dimensions and properties of the fluid and the structure are kept unchanged.

Benchmark CFD-LES.

In order to initialize the coupled FSI-LES case and to check the LES prediction of the turbulent flow, in a first step the flexible structure is again assumed to be rigid as in the laminar case. Thus, the FSI-LES benchmark reduces to a pure CFD test case denoted CFD-LES.

\section{Numerical Setup}

The predictions are carried out based on the numerical schemes described in $\S 2$ and $\S 3$. In the following specific settings are provided for both, laminar and turbulent cases separately.

\subsection{FSI Benchmark for Laminar Flow including CFD3 and CSM1-3}

In order to allow a grid independence study for the laminar flow cases CFD3 and FSI3, a series of three block-structured grids was taken into account for the flow predictions. In the cross-section the grid is successively refined by a factor of two in both directions. The coarsest grid (2-D) depicted in Fig. 6 consists of about 27,000 control volumes (CVs). The next finer level, i.e. the medium grid, possesses about 90,000 CVs and the fine grid consists of about 360,000 CVs. In wall-normal direction the first cell center is located at a distance of $\Delta y / D=6.925 \cdot 10^{-3}, 3.125 \cdot 10^{-3}$ and $1.5 \cdot 10^{-3}$ from the flexible structure for the coarse, medium and fine grid, respectively. Owing to the grid stretching applied in wall-normal direction, the ratio between two successive grid levels is not exactly one half. For the structure the corresponding grid is kept fixed for all three FSI3 predictions (coarse, medium and fine). It consists in total of 30 four-node shell elements. The same discretization is also applied for the preliminary structural tests CSM1 to CSM3.

Since for low Reynolds numbers the stability of explicit time-marching schemes is dominated by viscous effects, the time-step size has to be reduced with the refinement of the grid in order to stay in the stable limits ${ }^{2}$. Therefore, time-step sizes of $\Delta t_{f}=10^{-4} \mathrm{~s}$, (coarse grid), $5 \cdot 10^{-5} \mathrm{~s}$

\footnotetext{
${ }^{2}$ Since the FSI coupling scheme is mainly intended for high-Re flows within the LES context, this restriction solely applies to the present laminar test case.
} 
(medium grid) and $10^{-5} \mathrm{~s}$ (fine grid) are chosen to predict the flow field, respectively. Although in general different time-step sizes are possible for both solvers, presently this option is not implemented in the coupling tool CoMA. Hence in all cases considered the time-step size of the solver for the structure is adapted to the time-step size of the fluid solver, i.e., $\Delta t_{s}=\Delta t_{f}$. For time-stepping the structural solution the generalized $-\alpha$ method is applied with a spectral radius $\varrho_{\infty}=0.8$.

Within the coupled FSI prediction a constant underrelaxation factor of $\omega=0.5$ is considered for transferring the computed displacements from the structure domain to the fluid domain (see discussion in $\S 6.1 .4$ ). No underrelaxation of the fluid loads is necessary as mentioned in $\S 3.2$. For this case, a linear first-order extrapolation (11) for the estimation of the structural displacements at the beginning of each time step is performed. The choice is already motivated in $\S 3.2$.

FSI convergence is reached if the $\mathrm{L}_{2}$ norm of the displacement differences according to eq. (10) drops below $\varepsilon_{F S I}=10^{-5}$. For that purpose in the mean about 10 FSI-subiterations are required. Tests were also carried out with a limit of $\varepsilon_{F S I}=10^{-4}$ and $10^{-6}$. For the former reasonable results but partially overlayed by minor perturbations on the lift and drag curves were obtained. For the latter the same results as for $\varepsilon_{F S I}=10^{-5}$ were noticed which motivates the current choice.

Finally, it should be noted that the present coupling algorithm was also tested as a loosely coupled scheme by setting the number of FSI-subiterations to zero. However, as expected the scheme becomes unstable after a few time steps owing to the artificial added-mass effect, see, e.g., Causin et al. (2005); Förster et al. (2007); Förster (2007).

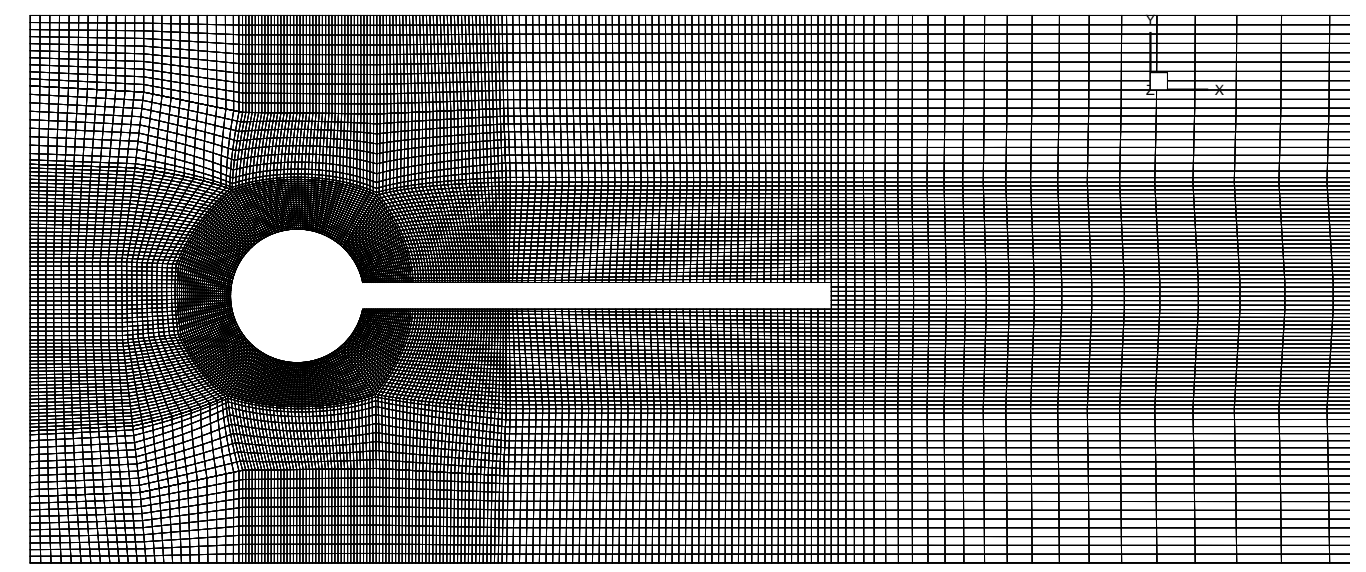

Figure 6: Coarse grid for the FSI3 test case in the $\mathrm{x}-\mathrm{y}$ cross-section, about half of the computational domain in the wake region omitted.

\subsection{FSI Benchmark for Turbulent Flow including CFD-LES}

For the flow prediction based on LES (i.e., the cases CFD-LES and FSI-LES) a blockstructured grid with about 17 million CVs is used, whereas $48 \mathrm{CVs}$ are applied in the spanwise direction. The grid points are clustered towards the rigid cylinder and the flexible structure using geometric stretching. The stretching factors are kept below 1.1 with the first cell center located at a distance of $\Delta y / D=1.5 \cdot 10^{-3}$ from the flexible structure. The prediction CFDLES for a rigid structure served as a source for the determination of time-averaged wall shear stresses on the cylinder and the structure. Based on that the average $y^{+}$values are predicted 
on the structure and found to be below 1 , mostly even below 0.2 . Thus, the viscous sublayer is adequately resolved. In contrast to the former case no grid clustering is required at the slip channel walls. The elastic plate is resolved by the use of $10 \times 10$ four-node shell elements.

For the turbulent case the restrictions concerning the time-step size are less restrictive as forecasted above. Thus a time-step of $\Delta t_{f}=10^{-4} \mathrm{~s}$ is chosen and again the same time-step size is applied for the structural solver based on the generalized $-\alpha$ method with $\varrho_{\infty}=0.8$.

Again a constant underrelaxation factor of $\omega=0.5$ is considered for the displacements and the loads are transferred without underrelaxation. Based on the experiences with the second-order extrapolation in the laminar case, here solely the first-order extrapolation for the estimation of the structural displacements at the beginning of each time step is used. In accordance with the laminar case the FSI convergence criterion is set to $\varepsilon_{F S I}=10^{-5}$ for the $\mathrm{L}_{2}$ norm of the displacement differences according to eq. (10).

For the LES predictions the national supercomputer SGI ALTIX $4700{ }^{3}$ was used applying in total 142 processors for the CFD part plus one processor for the coupling code and the CSD code, respectively.

\section{Results}

\subsection{Validation of the Methodology based on Laminar Flow}

In the following, first the results of the validation procedure for both subtasks (CFD and CSM) are briefly shown. Then the coupled laminar case FSI3 is discussed in more detail.

\subsubsection{Benchmark CFD3}

As mentioned above, replacing the flexible structure in Fig. 5 by a rigid plate, the FSI3 benchmark reduces to the pure CFD test case CFD3 (Turek and Hron, 2006). It considers the laminar flow around a cylinder with a splitter plate at the backside. The comparison will be done for fully developed flow, and particularly for one full period of the oscillation. Forces exerted by the fluid on the whole submerged body, i.e. lift and drag forces acting on the cylinder and the beam structure together, are taken into account for comparison. Although unusual, in order to be consistent with the reference data (Turek and Hron, 2006) provided by a fully implicit FEM method with a coupled multigrid solver, the results are provided by dimensional quantities. Furthermore, the forces are given per unit length since the problem is two-dimensional.

Despite the splitter plate behind the cylinder, vortex shedding occurs at $R e=200$. The shed vortices travel downstream and interact with the structure (not shown here, see similar behavior for the flexible structure in Fig. 7). The time history of the lift force shows a sinusoidal signal where the mean value is not equal to zero due to the slightly off-centered position of the structure in the channel. To quantify the results for comparison purposes, the minimal and maximal values are provided in Table 1 for the three successive grids applied. It is obvious that the values of the minimal and the maximal lift converge step by step towards the reference data when the grid is refined consecutively. Regarding the corresponding frequencies of the oscillating lift force the predicted values are about 1\% higher than in the reference case and that does not change with grid refinement. Since the scheme applied is second-order accurate in time and the time-step sizes are 50 to 500 times smaller than in the reference case, this minor deviation is still astonishing. As usual for the vortex shedding phenomenon past bluff bodies, the frequency of the drag force is doubled with respect to the lift force. Besides the absolute minimum and maximum the time history of the drag (not shown here) depicts a local

${ }^{3}$ http://www.lrz.de/services/compute/hlrb/ 
Table 1: Results of the CFD3 benchmark.

\begin{tabular}{llllllc} 
& $\begin{array}{l}\text { Resolution } \\
{\left[\times 10^{3} \mathrm{CVs}\right]}\end{array}$ & \multicolumn{2}{c}{ Drag $[\mathrm{N}]$} & \multicolumn{2}{c}{ Lift $[\mathrm{N}]$} & \multicolumn{2}{c}{ Frequency } \\
\cline { 3 - 6 } & Min. & Max. & Min. & Max. & of Lift $[\mathrm{Hz}]$ \\
\hline Coarse & 27 & 437 & 447 & -427 & 397 & 4.439 \\
\hline Medium & 90 & 435 & 446 & -448 & 421 & 4.443 \\
\hline Fine & 360 & 434 & 445 & -451 & 426 & 4.444 \\
\hline \hline Turek and Hron (2006) & highly resolved FE & 434 & 445 & -450 & 426 & 4.396
\end{tabular}

minimum and a local maximum. Table 1 provides the values of the absolute extrema. Again it is observed that the values of the present predictions converge towards the reference data when the grid is refined. Consequently, the grid refinement study was successful and the CFD solver is validated.

\subsubsection{Benchmarks CSM1 to CSM3}

The next step is to validate the CSD tool in an uncoupled environment using the benchmarks CSM1 to CSM3 suggested by Turek and Hron (2006). The setup solely includes the elastic beam without the surrounding fluid. The tests CSM1 and CSM2 are steady-state cases, whereas CSM3 has to be computed as a time-dependent case starting from the undeformed configuration. Tables 2 and 3 summarize the predicted steady-state deflections in both directions in comparison with the reference data (Turek and Hron, 2006). In all cases an error of less than $0.6 \%$ is found. Table 4 summarizes the comparison for the unsteady case CSM3 based on the minimal and maximal deflections and the frequency of the oscillation. A maximum deviation of about $1.1 \%$ is observed for the deflection in $\mathrm{x}$-direction. The minimum deflection in y-direction fits well to the reference data, whereas for the maximal deflection a value larger than the initial position is provided in (Turek and Hron, 2006) which is hard to explain. The predicted frequency is in close agreement with the reference data. In conclusion, based on minor deviations observed also the CSD solver is successfully validated.

Table 2: Results of the CSM1 benchmark.

\begin{tabular}{lll} 
& $d_{x}\left[\times 10^{-3} \mathrm{~m}\right]$ & $d_{y}\left[\times 10^{-3} \mathrm{~m}\right]$ \\
\hline present & -7.187 & -66.10 \\
\hline Turek and Hron $(2006)$ & -7.144 & -65.90 \\
\hline \hline Error & $0.598 \%$ & $0.303 \%$
\end{tabular}

\subsubsection{Benchmark FSI3}

The natural extension of the previous case is to release the flexible structure again and to tackle the coupled FSI problem. Fig. 7 depicts contours of the pressure and the streamwise velocity at an arbitrarily chosen time instant. Similar as before vortex shedding is observed at the cylinder ${ }^{4}$. Vortices detach from the cylinder in an alternating manner. They travel

\footnotetext{
${ }^{4}$ See FSI3 videos in the online version of the paper!
} 
Table 3: Results of the CSM2 benchmark.

\begin{tabular}{lll} 
& $d_{x}\left[\times 10^{-3} \mathrm{~m}\right]$ & $d_{y}\left[\times 10^{-3} \mathrm{~m}\right]$ \\
\hline present & -0.4690 & -16.97 \\
\hline Turek and Hron $(2006)$ & -0.4663 & -16.92 \\
\hline \hline Error & $0.576 \%$ & $0.295 \%$
\end{tabular}

Table 4: Results of the CSM3 benchmark.

\begin{tabular}{llllll} 
& \multicolumn{2}{c}{$d_{x}\left[\times 10^{-3} \mathrm{~m}\right]$} & \multicolumn{2}{c}{$d_{y}\left[\times 10^{-3} \mathrm{~m}\right]$} & Frequency \\
\cline { 2 - 6 } & Min. & Max. & Min. & Max. & {$[\mathrm{Hz}]$} \\
\hline present & -28.934 & 0.0 & -128.670 & $\approx 10^{-4}$ & 1.0980 \\
\hline Turek and Hron $(2006)$ & -28.610 & 0.0 & -128.767 & 1.553 & 1.0995 \\
\hline \hline Error & $1.132 \%$ & $0.0 \%$ & $0.075 \%$ & - & $0.13 \%$
\end{tabular}

downstream and start to interact with the flexible structure leading to an oscillating structure. In Fig. 7 the unbalanced pressure distribution on both sides of the flexible structure as the source for the oscillations as well as the shedding phenomenon are clearly visible.

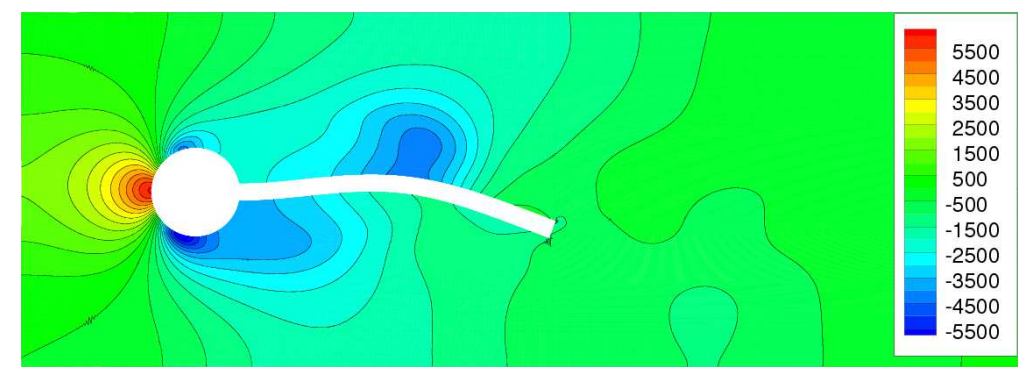

(a) Pressure

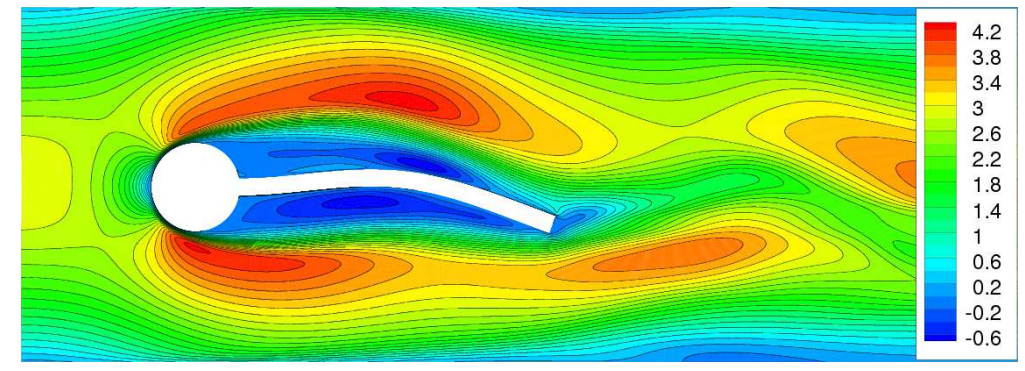

(b) Streamwise velocity

Figure 7: Instantaneous flow field for benchmark FSI3 (fine grid).

The time history of the lift force on the entire structure is displayed in Fig. 8. After a transition phase the amplitudes of the oscillation reaches constant values which are again asymmetric around the zero axis due to the asymmetry of the configuration. The values of the extrema obtained on the three grid levels are summarized in Table 5. It is obvious that the agreement with the reference data (Turek et al., 2010) provided by a monolithic, fully implicit ALE- 
Table 5: Results of the FSI3 benchmark.

\begin{tabular}{|c|c|c|c|c|c|c|}
\hline & \multirow{2}{*}{$\begin{array}{l}\text { Resolution } \\
{\left[\times 10^{3} \mathrm{CVs}\right]}\end{array}$} & \multicolumn{2}{|c|}{ Drag $[N]$} & \multicolumn{2}{|c|}{ Lift $[N]$} & \multirow{2}{*}{$\begin{array}{l}\text { Frequency } \\
\text { of Lift }[\mathrm{Hz}]\end{array}$} \\
\hline & & Min. & Max. & Min. & Max. & \\
\hline Coarse & 27 & 427.7 & 526.2 & -184.7 & 196.9 & 5.190 \\
\hline Medium & 90 & 423.9 & 515.4 & -172.8 & 184.3 & 5.165 \\
\hline Fine & 360 & 424.0 & 505.0 & -160.0 & 172.0 & 5.164 \\
\hline Turek et al. (2010) & monolithic FE & 432.7 & 488.2 & 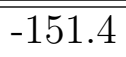 & 156.4 & 5.46 \\
\hline Gallinger (2010) & partitioned FV-FE & 446.8 & 503.0 & -162.0 & 169.8 & 5.51 \\
\hline
\end{tabular}

FEM method with a coupled multigrid solver, strongly improves when the grid is refined. Nevertheless, on the finest grid level a deviation with respect to Turek et al. (2010) remains. Concerning the frequency of the lift force the results obtained on the different grid levels are nearly constant and thus do not converge towards the data in Turek et al. (2010). A deviation of about $5.4 \%$ remains.

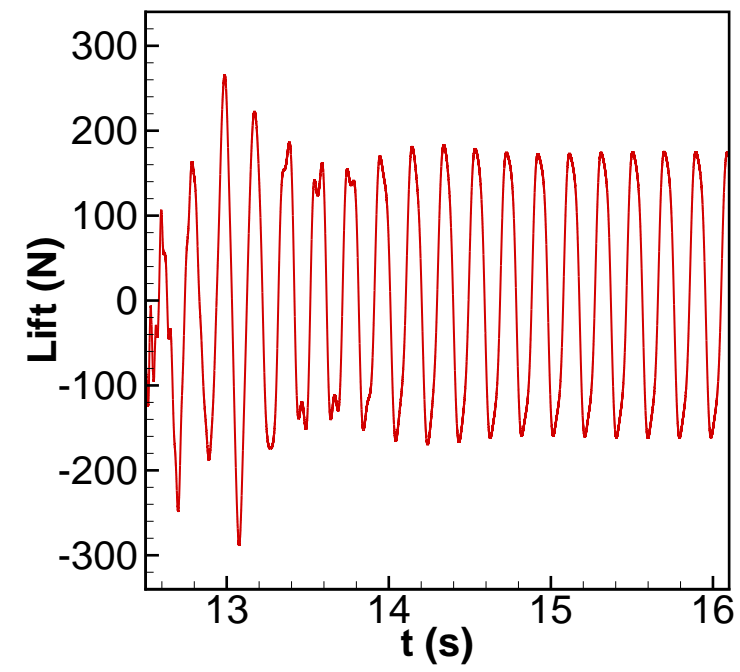

(a) Full scale

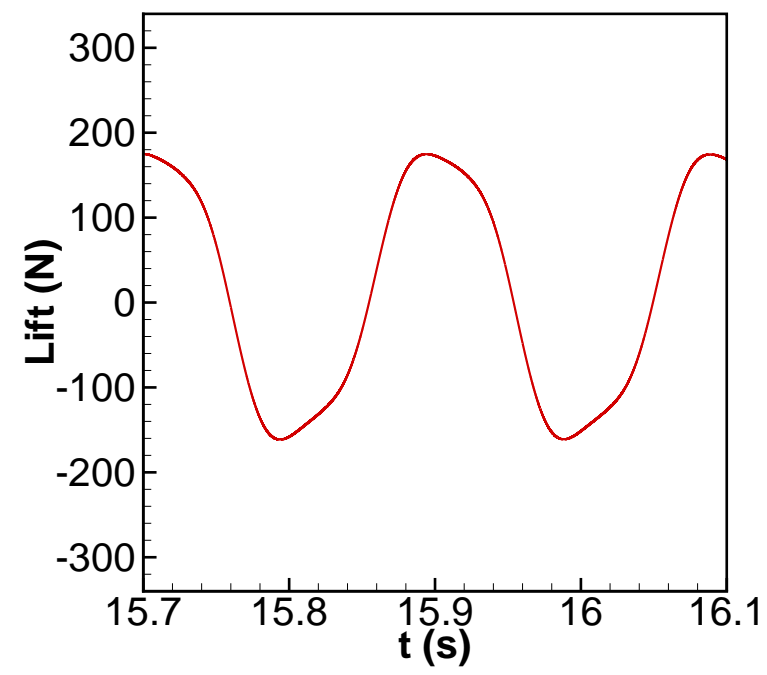

(b) Zoom

Figure 8: Time history of the lift force for benchmark FSI3 (fine grid).

With respect to the drag force (see Fig. 9) the present results are in closer agreement with the reference data than the lift. When refining the grid the extrema converge towards the data in Turek et al. (2010) and on the finest grid level a deviation of about 3.5\% or less is noticed.

In Bungartz et al. (2010) the paper by Turek et al. (2010) also summarizes results of a variety of different groups applying a wide spectrum of various numerical schemes, coupling conditions, resolutions and convergence criteria. It can be observed that the mean values and amplitudes of the lift and drag forces show a wide spreading of the results. Somehow that has to be expected since a highly non-linear and complex case is considered. Exemplarily, the results of Gallinger $(2010)^{5}$ are included in Table 5 . These predictions used for comparison rely on a partitioned

\footnotetext{
${ }^{5}$ Method also published in Hojjat et al. (2010) and results also available in Turek et al. (2010)!
} 
coupling scheme. The open source cell-based finite-volume solver OpenFOAM applies an implicit backward-differencing time-marching scheme, the PISO pressure-correction algorithm and the ALE formulation for the solution of the incompressible Navier-Stokes equations. That is combined with the same CSD solver and coupling tool as used in the present investigation, i.e., the finite-element solver Carat++ and CoMA, respectively. Furthermore, a similar resolution as in the present study is applied. Hence a comparison between both predictions is appreciated. Owing to the implicit time-stepping scheme a 5 to 50 times larger time-step size than in the present case was used. Furthermore, a fully implicit partitioned coupling algorithm based on fixed-point iterations with Aitken relaxation or a quasi-Newton method was applied in Gallinger (2010). For more details, please refer to Gallinger (2010) and Hojjat et al. (2010). Concerning the lift forces a very good level of agreement with the additional reference data (Gallinger, 2010) is achieved. However, for the drag force a better agreement regarding the mean value is observed in the present study, whereas the frequency of the lift is predicted in closer accordance with Turek et al. (2010) by Gallinger (2010).

By performing this test case the proper behavior of the whole partitioned FSI setup is proven. Despite some differences the overall agreement is satisfactory.

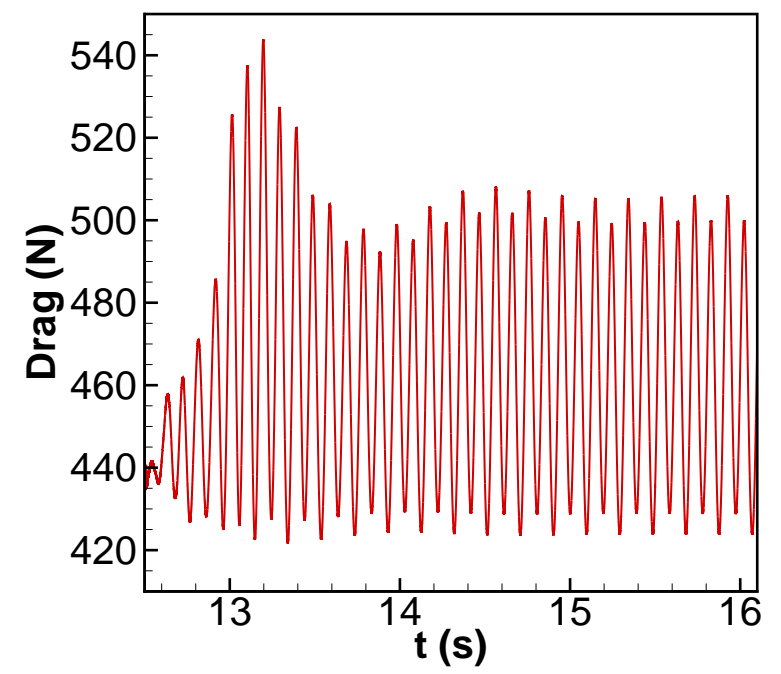

(a) Full scale

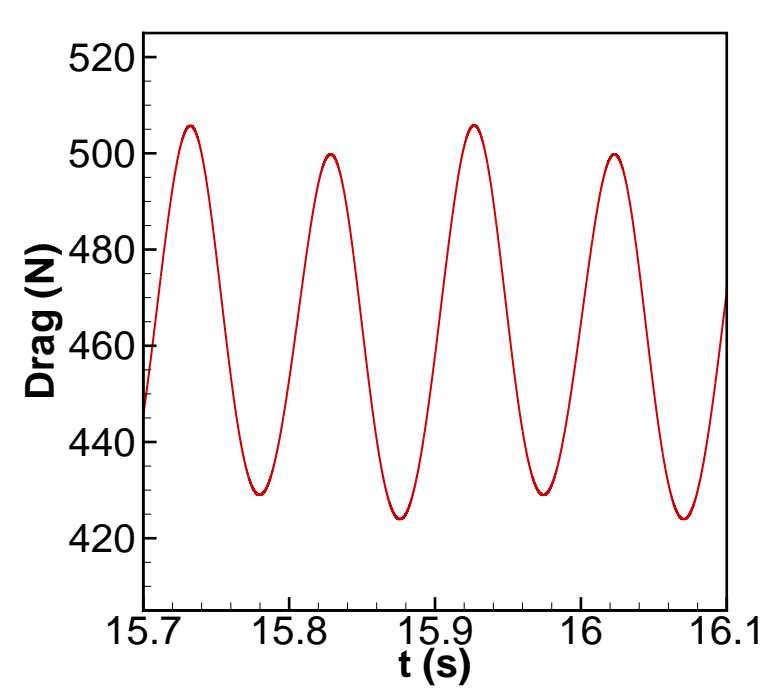

(b) Zoom

Figure 9: Time history of the drag force for benchmark FSI3 (fine grid).

\subsubsection{Comparison of Fixed and Dynamic Underrelaxation Factors}

As described in $\S 3.2$ the structural deformations predicted by the CSD solver are underrelaxed before the grid adaptation is carried out within the CFD code. For that purpose fixed or dynamically predicted values of the underrelaxation factor $\omega$ can be applied in eq. (13). In order to investigate the convergence behavior of the newly developed coupling scheme for different options concerning the underrelaxation factor, the FSI3 benchmark on the coarse grid is studied in detail. For these tests again the linear version (11) of the displacement estimator is used since no significant differences were found between both options described. Based on 40,000 time steps and the usual convergence criterion (i.e., $\mathrm{L}_{2}$ norm of the displacement differences $<\varepsilon_{F S I}=10^{-5}$ ) the mean value of the required number of FSI subiterations is considered. Table 6 summarizes the results for four fixed values in the range $0.1 \leq \omega \leq 0.6$ and two slightly different Aitken methods. The standard Aitken version relies on the formulation 
of Irons and Tuck (1969) and Mok (2001). The special version consists of the Aitken method by Mok (2001) but applied just in every second FSI-subiteration as suggested by Macleod (1986). Otherwise, a fixed value of $\omega=1$ is applied.

\begin{tabular}{l|c|c|c|c|c|c} 
& $\omega=0.1$ & $\omega=0.2$ & $\omega=0.5$ & $\omega=0.6$ & Aitken & Aitken Special \\
\hline \hline Refs. & & & & & $\begin{array}{c}\text { Aitken (1926) } \\
\text { Irons and Tuck (1969) } \\
\text { Mok (2001) }\end{array}$ & $\begin{array}{c}\text { Macleod (1986) } \\
\text { Mok (2001) }\end{array}$ \\
\hline$\#$ iter. & 55.21 & 29.51 & 9.38 & 14.00 & 9.48 & 8.85 \\
\hline $\begin{array}{l}\text { Comments } \\
\text { about } \\
\begin{array}{l}\text { history of } \\
\text { forces }\end{array}\end{array}$ & smooth & smooth & smooth & perturb. & perturbations & perturbations \\
\hline
\end{tabular}

Table 6: Comparison between different coupling algorithms; the FSI iteration number is the mean value of the complete simulation on the coarse grid.

As expected, small (fixed) values of $\omega$ lead to convergence but the number of subiterations required is quite large. By increasing $\omega$ up to about 0.5 the number of iterations until the convergence criterion is reached decreases to less than 10 . However, a further mild increase towards $\omega=0.6$ already yields to a worse convergence behavior and thus more iterations. Interestingly, the deterioration of the convergence is accompanied by visible perturbations of the time histories of the lift and drag forces. Although the convergence criterion is reached, these curves are no longer smooth but show minor perturbations which are superimposed on the signal. The same applies for both versions of the Aitken method. Based on the standard Aitken version the mean number of required subiterations is very similar to the version with a fixed value of $\omega=0.5$. However, in contrast to the fixed version the dynamic variant shows perturbations on the signal of the forces depicted in Fig. 10. Regarding the number of subiterations astonishingly the special Aitken method with a recomputation of $\omega$ every second iteration is the best choice, but again the resulting signals are not smooth. Reducing the convergence criterion to a lower value, e.g., $\varepsilon_{F S I}=10^{-7}$, the perturbations observed disappear for all underrelaxation factors and both Aitken schemes. However, a higher number of iterations is required to reach smooth curves, which is an obvious disadvantage.

Owing to the minor differences in the number of necessary iterations and the smooth signals, presently the version with fixed $\omega$ values found by numerical experiments is favored. Nevertheless, this issue requires further detailed investigations since in contrast to the present semi-implicit scheme fully implicit coupling schemes have shown the superiority of the adaptive stabilization, see, e.g., Wüchner (2006); Wüchner et al. (2007); Kupzok (2009); Küttler (2009); Degroote et al. (2008). One explanation might be the differences in the time-marching scheme and time-step size (c.f. discussion on stability in Förster et al. (2007) and Förster (2007)), which is much smaller in the present algorithm designed to resolve turbulent fluid flow in time.

\subsection{Turbulent Cases using LES}

First the results of the LES prediction for the rigid structure (CFD-LES) are discussed. Subsequently, the case of the principal objective of the present investigation, i.e., the turbulent 


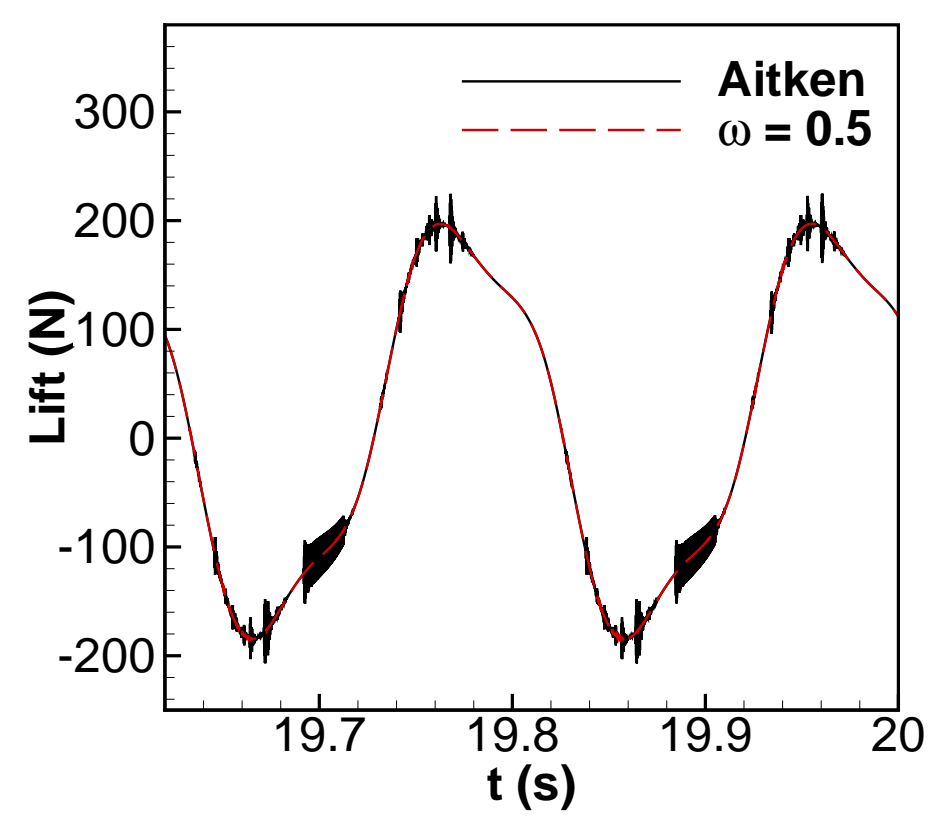

Figure 10: Comparison of the time history of the drag force for the Aitken scheme and a constant relaxation parameter $\omega=0.5$, convergence criterion: $\mathrm{L}_{2}$ norm of the displacement differences $<\varepsilon_{F S I}=10^{-5}$ (see eq. (10)).

FSI case (FSI-LES) is considered. In the following all results are presented in dimensionless manner using $u_{\infty}$ and $D$ as reference quantities.

\subsubsection{Rigid Structure: CFD-LES}

For the turbulent case, the simulation was started again with a rigid structure, thus a pure LES without coupling. For the flow around a cylinder a variety of experimental as well as numerical investigations are available, see, e.g., Breuer (1998, 2000, 2002). Owing to remarkably complex flow features such as thin separating shear layers, transition and large-scale vortex motion in the wake, the cylinder flow can be considered as the paradigm of complex flows and thus as a challenging test case for flow predictions. At the present Reynolds number of $R e=10^{4}$ the flow is still sub-critical, i.e. the boundary layers at the cylinder separate laminarly and transition takes place in the free shear layers induced by a Kelvin-Helmholtz instability. In the wake strong vortex shedding is observed for the free cylinder flow.

In the present case the structure behind the cylinder acts like a splitter plate of length $\mathrm{l} / \mathrm{D}=3.5$ attenuating the generation of a vortex street behind the cylinder. Nevertheless, quasi-periodic vortex shedding is still observed. Fig. 11 depicts an arbitrarily chosen snapshot of the distribution of the pressure and the streamwise velocity in a $\mathrm{x}-\mathrm{y}$ plane showing the shear layers with the Kelvin-Helmholtz instability leading to transition and large vortices originating from the shedding process ${ }^{6}$. The time history of the lift and drag coefficients depicted in Fig. 12 confirms that the splitter plate does not suppress vortex shedding completely. The lift coefficient strongly oscillates around a slightly positive mean values with amplitudes of about \pm 2 . These are significantly larger than observed for the pure cylinder flow (Breuer, 1998, 2000, 2002). The cause for this observation is the splitter plate since the pressure distributions on both sides

\footnotetext{
${ }^{6}$ See CFD-LES videos in the online version of the paper!
} 
significantly contribute to the resulting lift forces on the entire structure. The drag coefficient shows much lower amplitudes and varies around a mean values of about 1.2 which is in the range of values expected for the single cylinder in this Reynolds number range. Thus the drag is much less influenced by the splitter plate than the lift coefficient. Since the surface of the splitter plate is oriented in the direction of the main flow, this outcome has to be expected. Except on the rear panel solely the shear forces on the splitter plate contribute to the drag force and since these are comparably small $(\approx 1 \%$ of the total force), the effect of the splitter plate is minor.

Compared to a pure cylinder in free flow the Strouhal number decreases from about St $=0.2$ to $\mathrm{St}=0.175$ which is the result of two opposing effects: the splitter plate typically decreases the Strouhal number, while due to the blockage effect of the channel St is slightly increased again.

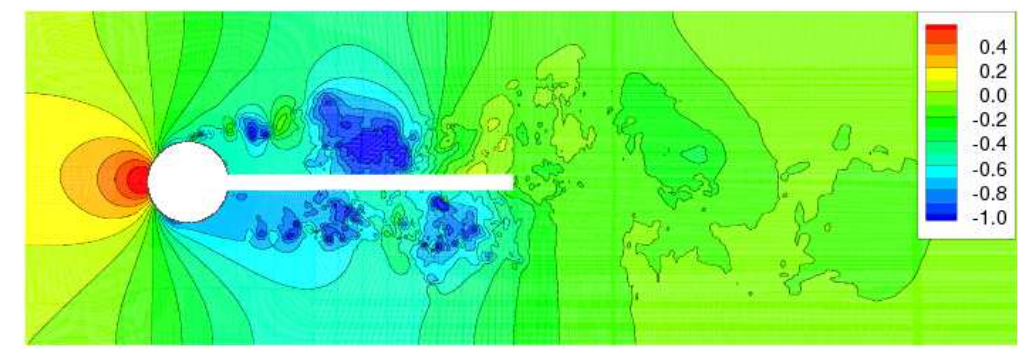

(a) Pressure

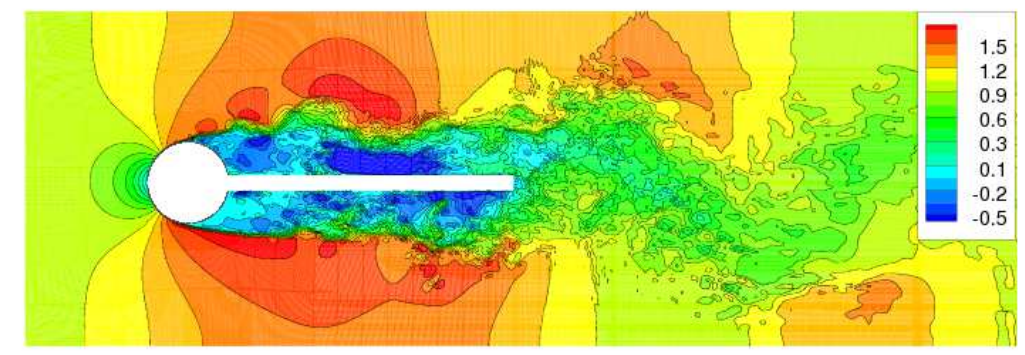

(b) Streamwise velocity

Figure 11: Instantaneous flow field for the turbulent case with a rigid structure ( $\mathrm{x}-\mathrm{y}$ plane).

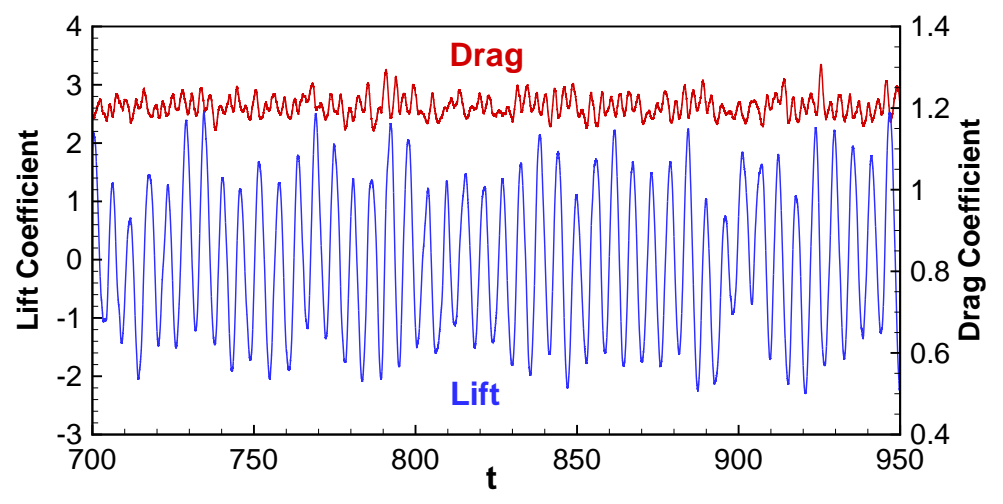

Figure 12: Time history of the lift and drag coefficient for the turbulent case with a rigid structure.

In order to further investigate the flow field, the instantaneous flow was averaged over a long 
time period of about 540 dimensionless time units ${ }^{7}$ which is equivalent to about 100 vortex shedding cycles. Fig. 13(a) displays the time-averaged flow by streamlines, whereas Fig. 13(b) depicts the distribution of the time-averaged velocity $\bar{u}$ in mainstream direction. The LES computation predicts an attached recirculation region behind the cylinder on both sides of the plate. The length of the recirculation region $\left(L_{r} / D \approx 3.25\right)$ is much longer than for cases without a splitter plate (Breuer, 1998, 2000, 2002) as expected. It ends shortly before the trailing edge of the plate, i.e., reattachment is observed at about $D / 4$ before the edge.

In addition to the mean flow field, higher-order moments have been investigated. In Figs. 13(c) to 13(e) the total resolved Reynolds stresses $\overline{u^{\prime} u^{\prime}}, \overline{v^{\prime} v^{\prime}}$ and $\overline{u^{\prime} v^{\prime}}$ are plotted ${ }^{8}$. Except the fact that due to the splitter plate the entire flow is somehow stretched in streamwise direction as already observed for the streamlines and $\bar{u}$, the distribution of the streamwise Reynolds stress $\overline{u^{\prime} u^{\prime}}$ as well as the shear stress $\overline{u^{\prime} v^{\prime}}$ are qualitatively in close agreement with data obtained for the single cylinder (Breuer, 1998, 2000, 2002). An exception represents the cross-stream Reynolds stress shown in Fig. 13(d). Whereas for the pure cylinder the maximum values are found on the symmetry axis owing to the vortex shedding motion, in the present case this maximum is intersected by the splitter plate. Furthermore, a second maximum develops behind the backside of the plate.

The distributions of the Reynolds stresses also confirm the statement that the flow is in the sub-critical regime. On the entire cylinder vanishing values are found. Hence the flow is laminar. Transition to turbulence occurs in the free shear layer as obvious from Figs. 13(c) to 13(e). Since also in the case of the flexible plate to be discussed in the next section, the cylinder will be kept rigid, the situation will not change for the coupled case.

\subsubsection{Flexible Structure: FSI-LES}

Starting from the LES prediction of the flow around the rigid structure (CFD-LES), the plate is released and a fully coupled FSI-LES prediction is started. Owing to different loads on both sides the structure directly starts to deflect in one direction. After a short initial phase, in which the amplitudes of the deflections successively increase, a new quasi-periodic mode of oscillation is reached $^{9}$. In Fig. 14 the time history of the lift and drag coefficients are depicted in the same manner as for the pure LES case (Fig. 12). At $t=20$ the coupled simulation is started. After about three initial oscillations a new dynamic state is visible. Compared to the rigid case the magnitudes of the lift coefficient are reduced to about $75 \%$, whereas neither for the amplitudes nor for the mean values of the drag coefficient a significant change is found. A FFT of the lift signal furthermore shows that the main frequency is dramatically reduced leading to a Strouhal number of $S t=0.103$ compared to 0.175 before.

In Figs. 15 and 16 a complete cycle of the structural oscillation is shown based on the distribution of the pressure and the streamwise velocity component in a $\mathrm{x}-\mathrm{y}$ plane. $T_{0}$ denotes the time instant when the plate is about in its original position and $\Delta T \approx 9.7$ stands for the time interval of a complete cycle. Similar to the uncoupled case the shear layers developing out of the separated boundary layers at the cylinder are visible. Furthermore, vortices with low pressure kernels are generated in these shear layers and travel downstream.

In the first snapshot (Fig. 15(a)) a large region of low pressure is visible at the upper side of the plate. That is the remainder of an even stronger pressure minimum observed about a quarter of an interval before (see, e.g., Fig. 15(d)). That represents the main cause for the bending

\footnotetext{
${ }^{7}$ Time normalized by $u_{\infty}$ and $D$.

${ }^{8}$ The overbar - denotes the time average and the prime $u^{\prime}$ stands for the fluctuating velocity component.

${ }^{9}$ See FSI-LES videos in the online version of the paper!
} 


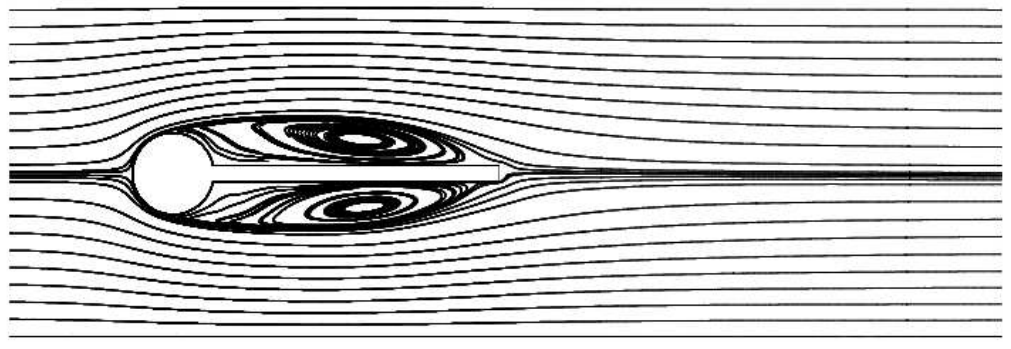

(a) Streamlines

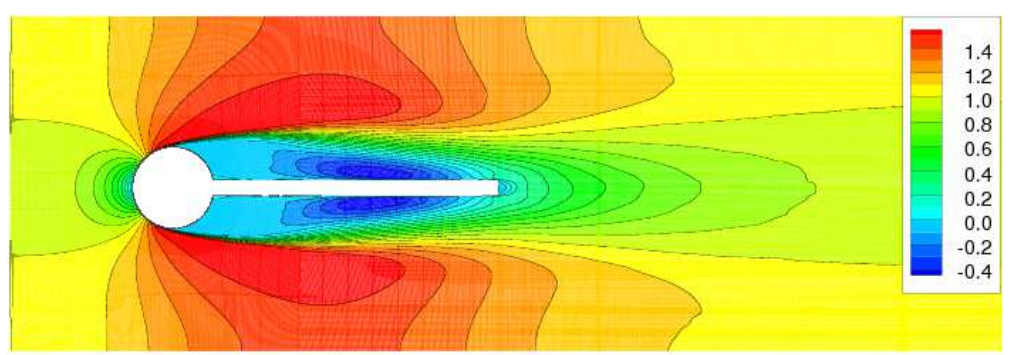

(b) Streamwise velocity $\bar{u}$

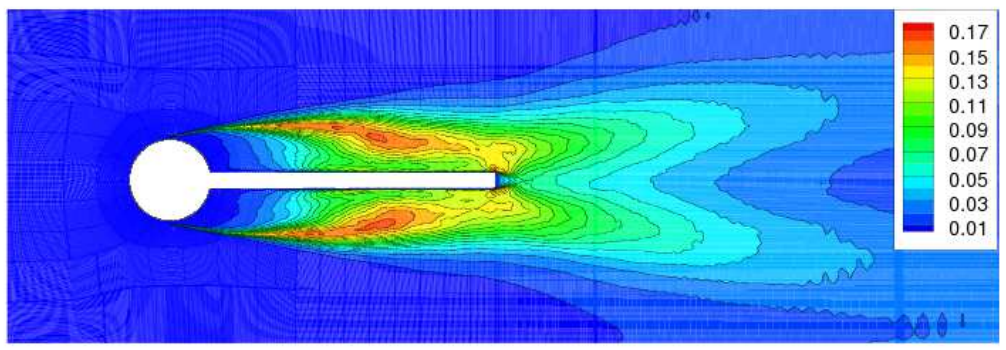

(c) Streamwise Reynolds stress $\overline{u^{\prime} u^{\prime}}$

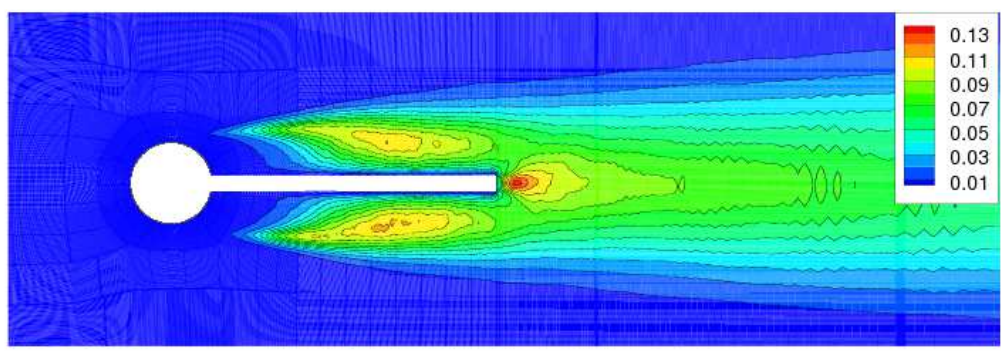

(d) Cross-stream Reynolds stress $\overline{v^{\prime} v^{\prime}}$

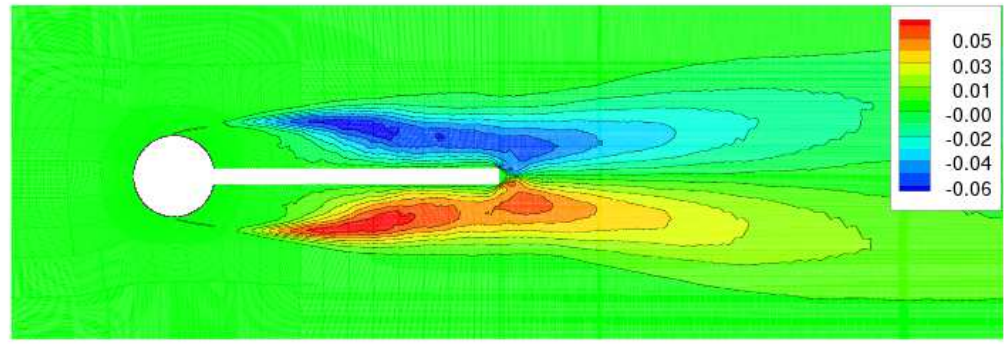

(e) Reynolds shear stress $\overline{u^{\prime} v^{\prime}}$

Figure 13: Time-averaged flow field for the turbulent case with a rigid structure.

motion noticeable in the second subfigure. At this instant the bending of the plate reaches about its maximum. Due to the bending of the structure the lower side of the structure forms a 


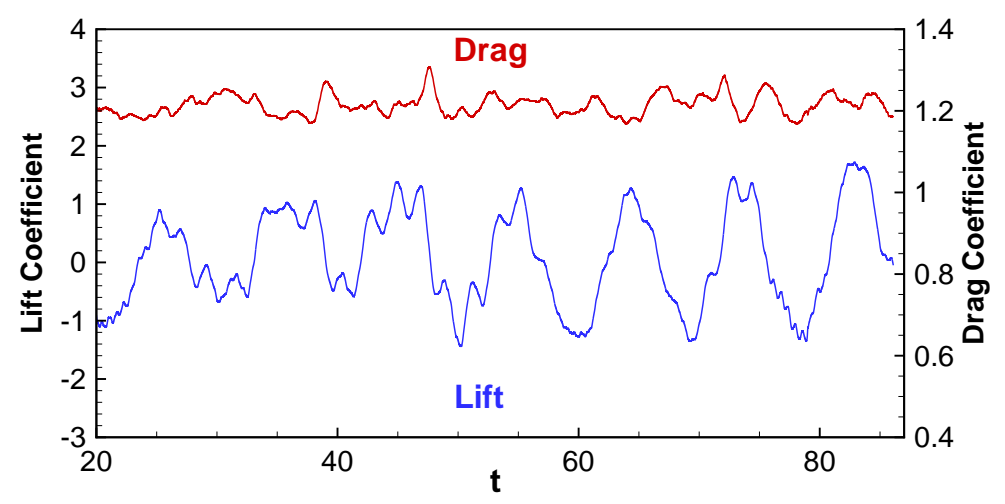

Figure 14: Time history of the lift and drag coefficient for the turbulent case with a flexible structure.

convex surface similar to an airfoil leading to a new pressure minimum on the lower side visible in Fig. 15(b). That is mainly responsible for the bending-back motion of the structure to its initial position depicted in Fig. 15(c). Then the entire process is continued mirror-inverted in the other direction but of course based on the same mechanism. The plate is deflected downwards and a low pressure region on the upper side is generated due to the convex surface of the plate on this side, see Fig. 15(d). Consequently, owing to inertia, a phase shift of about $\Delta T / 2$ between the appearance of the pressure minimum on the convex side and the occurrence of the maximal deflection is observed.

The deflection of the structure also influences the development of the wake region as visible in Figs. 16(a) to 16(d). The wake follows the bent structure as expected.

In Fig. 17 the deformed plate is shown in three-dimensions. Furthermore, a snapshot of isosurfaces of the pressure colored by the streamwise velocity component is depicted. Although the flow field strongly varies in spanwise direction, a bending of the flexible structure in spanwise direction is hardly visible. Thus the variation of the pressure and shear forces in this spatial direction are too weak to be noticed as resulting deflections.

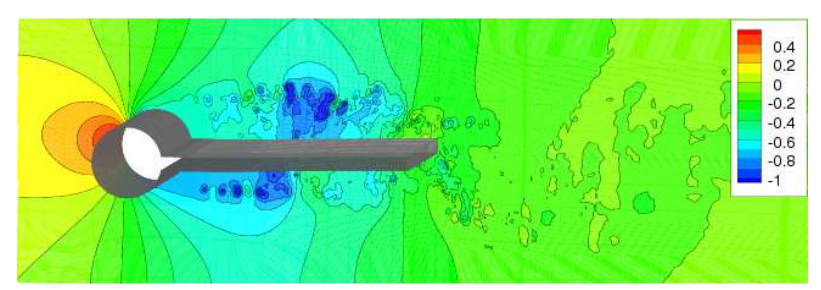

(a) $T_{0}$

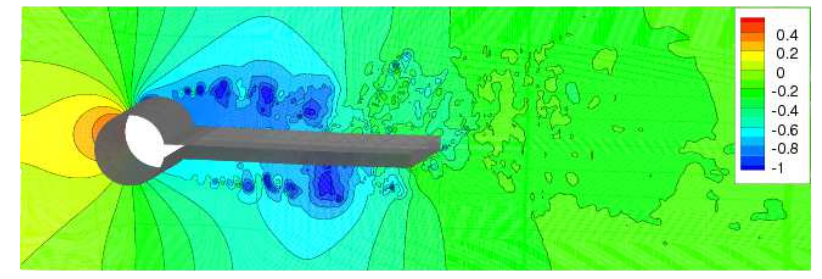

(c) $T_{0}+1 / 2 \Delta T$

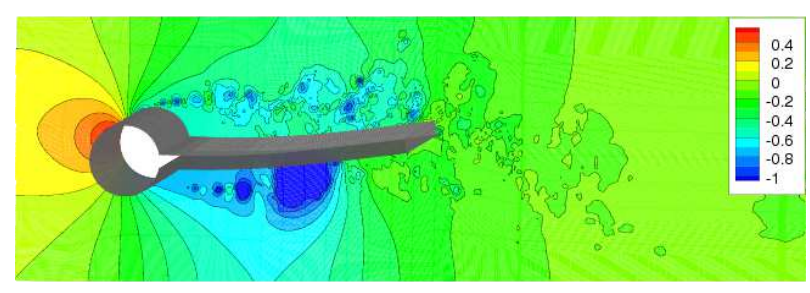

(b) $T_{0}+1 / 4 \Delta T$

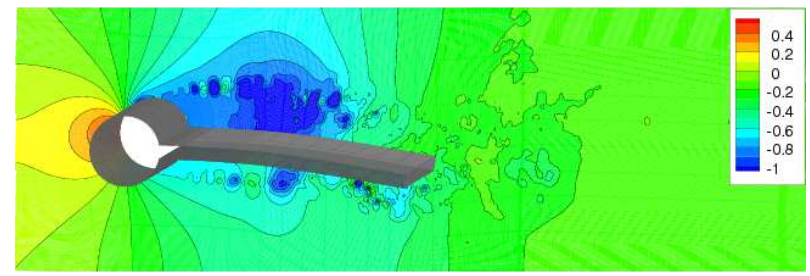

(d) $T_{0}+3 / 4 \Delta T$

Figure 15: Temporal development of the flow field and the structure for the turbulent case with a flexible structure, pressure distribution in a $\mathrm{x}-\mathrm{y}$ plane. 


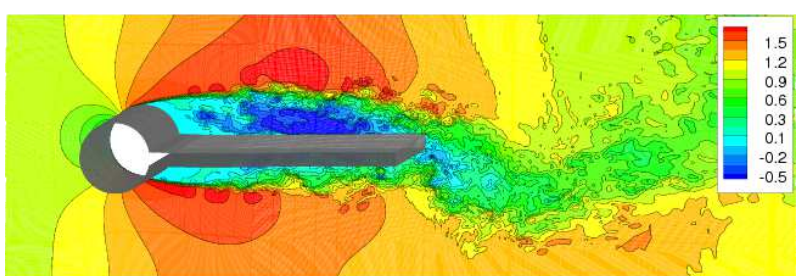

(a) $T_{0}$

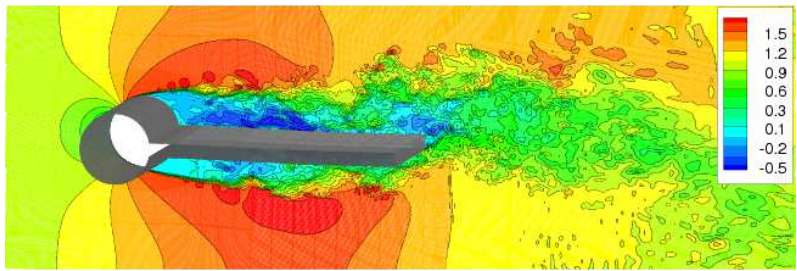

(c) $T_{0}+1 / 2 \Delta T$

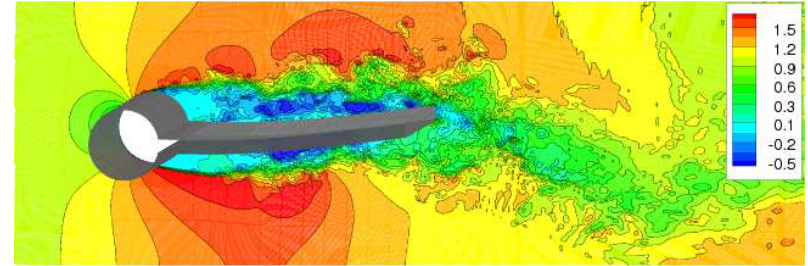

(b) $T_{0}+1 / 4 \Delta T$

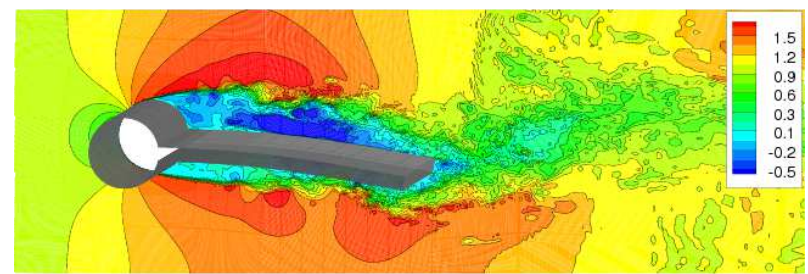

(d) $T_{0}+3 / 4 \Delta T$

Figure 16: Temporal development of the flow field and the structure for the turbulent case with a flexible structure, streamwise velocity distribution in a $\mathrm{x}-\mathrm{y}$ plane.

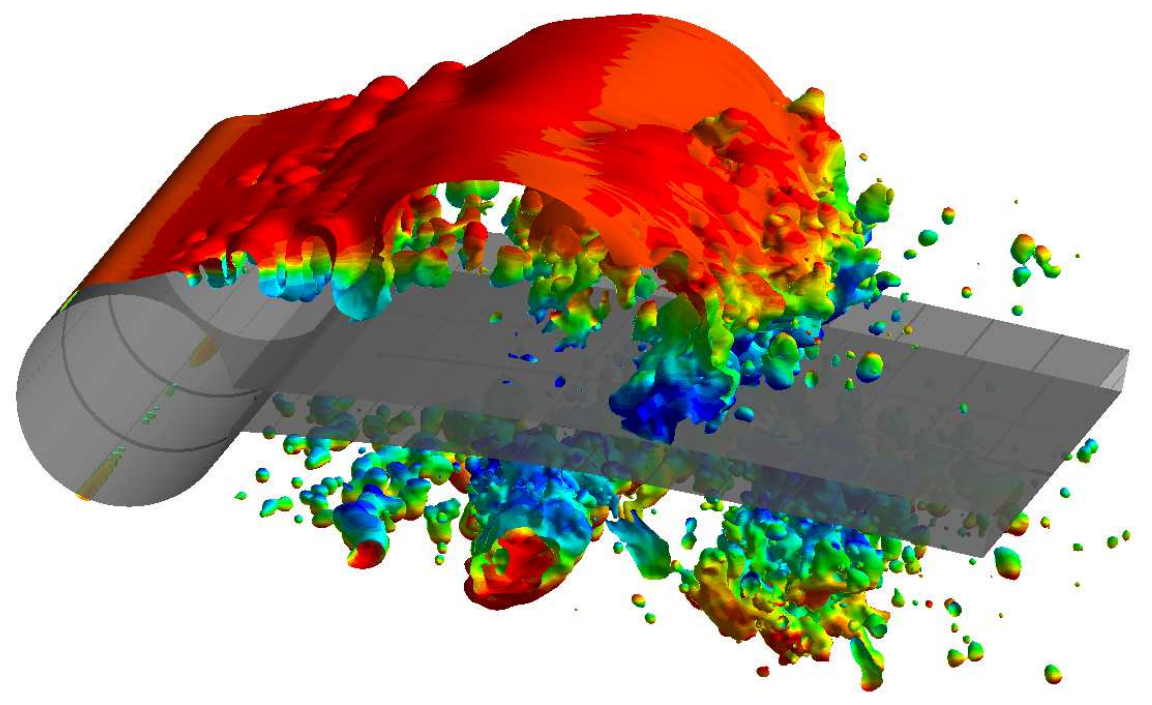

Figure 17: Snapshot of the turbulent flow around the flexible structure showing iso-surfaces of the pressure colored by the streamwise velocity component.

\section{Conclusions}

Since in many practically relevant fluid-structure interactions turbulence plays a dominant role, the time is ripe to tackle this problem with modern eddy-resolving schemes. Besides direct numerical simulation these are LES and with increasing potential also hybrid LES-RANS methods. The resolution of the large-scale structures in time typically requires small timestep size within the range of the stability limits of explicit schemes, e.g., predictor-corrector schemes. In the present study a partitioned coupling scheme for the marriage of FSI and LES (or related methods) is proposed which takes the specific needs of both disciplines into 
account, i.e., resolution in time and stability at high fluid-to-structure density ratios inducing the added-mass effect. The scheme involves an explicit solution of the non-linear terms in the Navier-Stokes equations and an implicit coupling between the computation of the pressure field and the displacements of the structure. Thus a repeated solution of expensive parts is avoided and a fast convergence of the FSI-subiterations is observed. The fluid solver is coupled to a structure solver especially designed for light thin-walled structures such as shells and membranes. Thus an appropriate tool for the time-resolved prediction of lightweight structures exposed to turbulent flows results.

For a thorough validation of the entire scheme, in a first step the solvers for each single fields were considered separately. For that purpose benchmark cases suggested in the literature were taken into account (CSM1-3 and CFD3). In a second step the entire methodology and the coupling scheme were investigated based on a fully coupled laminar benchmark case FSI3 including a grid independence study. The comparison yielded a close agreement with the reference data although the algorithms are totally different between the present study and the reference case. In a third step the study was extended into the turbulent regime starting with the prediction of the instantaneous and time-averaged flow field around the fixed rigid structure (CFD-LES). In a final step the coupling scheme was applied to the flexible structure in turbulent flow denoted FSI-LES. The resulting flow field and structural deflections were analyzed. The test cases clearly demonstrates that the proposed coupling scheme works in a robust and efficient manner, requiring only about 5 to 10 subiterations between the pressure and the displacement predictions to reach the FSI convergence criterion. The scheme is applicable to all other eddy-resolving schemes and the algorithm can be fully scheduled in parallel. Next steps will be the usage of improved grid adaptation schemes and eddy-resolving LES predictions of more complex FSI cases for lightweight structures (see, e.g., Wüchner (2006); Wüchner et al. (2007); Kupzok (2009); Gallinger (2010) for corresponding RANS predictions).

\section{Acknowledgments}

We gratefully acknowledge the cooperation with the Chair of Structural Analysis of TU Munich providing the codes Carat++ (Fischer et al., 2010; Bletzinger et al., 2006) and CoMA (Gallinger et al., 2009) including intensive support by Prof. Dr.-Ing. K.-U. Bletzinger. The computations were partially carried out on the German Federal Top-Level Computer SGI ALTIX at LRZ Munich under the contract number pr47me. All kinds of support are gratefully acknowledged.

\section{References}

Aitken, A. C., 1926. On Bernoulli's numerical solution of algebraic equations. In: Proceedings of the Royal Society of Edinburgh. Vol. 46. pp. 289-305.

Belytschko, T., Liu, W. K., Moran, B., 2000. Non-Linear Finite Elements For Continua and Structures. Vol. 36. John Wiley \& Sons Ltd, New York.

Bischoff, M., Wall, W. A., Bletzinger, K.-U., Ramm, E., 2004. Models and finite elements for thin-walled structures. In: Stein, E., De Borst, R., Hughes, T. J. R. (Eds.), Encyclopedia of Computational Mechanics. Vol. 2. John Wiley \& Sons Ltd, Chichester, pp. 59-138.

Bletzinger, K.-U., Wüchner, R., Kupzok, A., 2006. Algorithmic treatment of shells and free form-membranes in FSI. In: Bungartz, H.-J., Schäfer, M. (Eds.), Fluid-Structure Interaction. Vol. 53 of Lecture Notes in Computational Science and Engineering, LNCSE. Springer, Heidelberg, pp. 336-355. 
Breuer, M., 1998. Large-eddy simulation of the sub-critical flow past a circular cylinder: Numerical and modeling aspects. International Journal for Numerical Methods in Fluids 28 (9), 1281-1302.

Breuer, M., 2000. A challenging test case for large-eddy simulation: High Reynolds number circular cylinder flow. International Journal of Heat and Fluid Flow 21 (5), 648-654.

Breuer, M., 2002. Direkte Numerische Simulation und Large-Eddy Simulation turbulenter Strömungen auf Hochleistungsrechnern. Habilitationsschrift, Universität Erlangen-Nürnberg, Berichte aus der Strömungstechnik. Shaker Verlag, Aachen.

Breuer, M., Münsch, M., 2008. Fluids-structure interaction using LES - A partitioned coupled predictor-corrector scheme. Proc. in Applied Mathematics and Mechanics, PAMM 8 (1), 10515-10516.

Breuer, M., Münsch, M., 2010. LES meets FSI - important numerical and modeling aspects. In: Vincenzo, A., Geurts, B. J., Fröhlich, J. (Eds.), ERCOFTAC Series, Direct and Large-Eddy Simulation VII, 7th Int. ERCOFTAC Workshop on Direct and Large-Eddy Simulation: DLES-7, Sept. 8-10, 2008. Vol. 13. Springer Science+Business Media B.V., Trieste, Italy, pp. 245-251.

Bungartz, H.-J., Mehl, M., Schäfer, M. (Eds.), 2010. Fluid Structure Interaction II: Modelling, Simulation, Optimization. Vol. 73 of Lecture Notes in Computational Science and Engineering, LNCSE. Springer, Heidelberg.

Causin, P., Gerbeau, J.-F., Nobile, F., 2005. Added-mass effect in the design of partitioned algorithms for fluid-structure problems. Computer Methods in Applied Mechanics and Engineering $194(42-44)$.

Chorin, A. J., 1968. Numerical solution of the Navier-Stokes equations. Mathematics of Computations 22 (104), 745-762.

Chung, J., Hulbert, G. M., 1993. A time integration algorithm for structural dynamics with improved numerical dissipation: The generalized $-\alpha$ method. Journal of Applied Mechanics $60,371-375$.

Degroote, J., Bruggeman, P., Haelterman, R., Vierendeels, J., 2008. Stability of a coupling technique for partitioned solvers in FSI applications. Computers \& Structures 86 (23-24), $2224-2234$.

Demirdžić, I., Perić, M., 1988. Space conservation law in finite-volume calculations of fluid flow. International Journal for Numerical Methods in Fluids 8 (9), 1037-1050.

Demirdžić, I., Perić, M., 1990. Finite--volume method for prediction of fluid flow in arbitrarily shaped domains with moving boundaries. International Journal for Numerical Methods in Fluids $10(7), 771-790$.

Durst, F., Schäfer, M., 1996. A parallel block-structured multigrid method for the prediction of incompressible flows. International Journal for Numerical Methods in Fluids 22 (6), 549-565.

Durst, F., Schäfer, M., Wechsler, K., 1996. Efficient simulation of incompressible viscous flows on parallel computers. In: Hirschel, E. H. (Ed.), Flow Simulation with High-Performance Computers II, Notes on Numerical Fluid Mechanics. Vol. 52(1). Vieweg, pp. 87-101. 
Farhat, C., 2004. CFD-based non-linear computational aeroelasticity. In: Stein, E., De Borst, R., Hughes, T. J. R. (Eds.), Encyclopedia of Computational Mechanics. Vol. 3. John Wiley \& Sons Ltd, Chichester, pp. 459-477.

Farhat, C., Geuzaine, P., Grandmont, C., 2001. The discrete geometric conservation law and the non-linear stability of ALE schemes for the solution of flow problems on moving grids. Journal of Computational Physics 174 (2), 669-694.

Farhat, C., Lesoinne, M., LeTallec, P., 1998. Load and motion transfer algorithms for fluidstructure interaction problems with non-matching discrete interfaces: Momentum and energy conservation, optimal discretization and application to aeroelasticity. Computer Methods in Applied Mechanics and Engineering 157, 95-114.

Farhat, C., van der Zee, K. G., Geuzaine, P., 2006. Provably second-order time-accurate loosely-coupled solution algorithms for transient non-linear computational aeroelasticity. Computer Methods in Applied Mechanics and Engineering 195 (17-18), 1973-2001.

Felippa, C. A., Geers, T. L., 1988. Partitioned analysis for coupled mechanical systems. Engineering Computations 5, 123-133.

Felippa, C. A., Park, K. C., 1980. Staggered transient analysis procedures for coupled mechanical systems: Formulation. Computer Methods in Applied Mechanics and Engineering 24 (1), 61-111.

Fernández, M. A., Gerbeau, J. F., Grandmont, C., 2007. A projection semi-implicit scheme for the coupling of an elastic structure with an incompressible fluid. International Journal for Numerical Methods in Engineering 69, 794-821.

Fischer, M., Firl, M., Masching, H., Bletzinger, K.-U., 2010. Optimization of non-linear structures based on object-oriented parallel programming. In: Topping, B. H. V., Adam, J. M., Pallarés, F. J., Bru, R., Romero, M. L. (Eds.), Seventh Int. Conf. Engineering Computational Technology, ECT2010. Civil-Comp Press, Stirlingshire, UK, p. 67.

Förster, C., 2007. Robust methods for fluid-structure interaction with stabilised finite elements. Ph.D. thesis, Institut für Baustatik und Baudynamik, Universität Stuttgart, Germany.

Förster, C., Wall, W. A., Ramm, E., 2007. Artificial added mass instabilities in sequential staggered coupling of non-linear structures and incompressible viscous flows. Computer Methods in Applied Mechanics and Engineering 196 (7), 1278-1293.

Gallinger, T., 2010. Effiziente Algorithmen zur partitionierten Lösung stark gekoppelter Probleme der Fluid-Struktur-Wechselwirkung. Ph.D. thesis, Lehrstuhl für Statik, Technische Universität München, Germany.

Gallinger, T., Kupzok, A., Israel, U., Bletzinger, K.-U., Wüchner, R., 2009. A computational environment for membrane-wind interaction. In: Hartmann, S., Mesiter, A., Schäfer, M., Turek, S. (Eds.), International Workshop on Fluid-Structure Interaction: Theory, Numerics and Applications. Kassel University Press GmbH, pp. 283-294.

Germano, M., Piomelli, U., Moin, P., Cabot, W. H., 1991. A dynamic subgrid-scale eddy viscosity model. Physics of Fluids A 3, 1760-1765. 
Glück, M., Breuer, M., Durst, F., Halfmann, A., Rank, E., 2001. Computation of fluid-structure interaction on lightweight structures. Journal of Wind Engineering and Industrial Aerodynamics 89 (14-15), 1351-1368.

Glück, M., Breuer, M., Durst, F., Halfmann, A., Rank, E., 2003. Computation of wind-induced vibrations of flexible shells and membranous structures. Journal of Fluids and Structures 17 (5), 739-765.

Heil, M., 2004. An efficient solver for the fully coupled solution of large-displacement fluidstructure interaction problems. Computer Methods in Applied Mechanics and Engineering $193(1-2), 1-23$.

Hojjat, M., Stavropoulou, E., Gallinger, T., Israel, U., Wüchner, R., Bletzinger, K.-U., 2010. Fluid-structure interaction in the context of shape optimization and computational wind engineering. In: Bungartz, H.-J., Mehl, M., Schäfer, M. (Eds.), Fluid-Structure Interaction II - Modelling, Simulation, Optimization. Vol. 73 of Lecture Notes in Computational Science and Engineering, LNCSE. Springer, Heidelberg, pp. 351-381.

Irons, B. M., Tuck, R. C., 1969. A version of the Aitken accelerator for computer iteration. International Journal for Numerical Methods in Engineering 1 (3), 275-277.

Kordulla, W., Vinokur, M., 1983. Efficient computation of volume in flow predictions. AIAA Journal 21 (6), 917-918.

Kupzok, A. M., 2009. Modeling the interaction of wind and membrane structures by numerical simulation. Ph.D. thesis, Lehrstuhl für Statik, Technische Universität München, Germany.

Küttler, U., 2009. Effiziente Lösungsverfahren für Fluid-Struktur-Interaktions-Probleme. Ph.D. thesis, Lehrstuhl für Numerische Mechanik, Technische Universität München, Germany.

Küttler, U., Wall, W. A., 2008. Fixed-point fluid-structure interaction solvers with dynamic relaxation. Computational Mechanics 43 (1), 61-72.

Küttler, U., Wall, W. A., 2009. Vector extrapolation for strong coupling fluid-structure interaction solvers. Journal of Applied Mechanics 76, 021205.

Lesoinne, M., Farhat, C., 1996. Geometric conservation laws for flow problems with moving boundaries and deformable meshes, and their impact on aeroelastic computations. Computer Methods in Applied Mechanics and Engineering 134 (1-2), 71-90.

Lilly, D. K., 1992. A proposed modification of the Germano subgrid-scale closure method. Physics of Fluids A 4, 633-635.

Macleod, A. J., 1986. Acceleration of vector sequences by multi-dimensional $\Delta^{2}$ methods. Communications in Applied Numerical Methods 2 (4), 385-392.

Mok, D. P., 2001. Partitionierte Lösungsansätze in der Strukturdynamik und der FluidStruktur-Interaktion. Ph.D. thesis, Institut für Baustatik, Universität Stuttgart, Germany.

Münsch, M., Breuer, M., 2010. Numerical simulation of fluid-structure interaction using eddyresolving schemes. In: Bungartz, H.-J., Mehl, M., Schäfer, M. (Eds.), Fluid-Structure Interaction II - Modelling, Simulation, Optimization. Vol. 73 of Lecture Notes in Computational Science and Engineering, LNCSE. Springer, Heidelberg, pp. 221-253. 
Park, K. C., 1980. Partitioned transient analysis procedures for coupled field problems: Stability analysis. Journal of Applied Mechanics 47, 370-376.

Park, K. C., Felippa, C. A., 1983. Partitioned analysis of coupled systems. Computational Methods for Transient Analysis, 157-219.

Park, K. C., Felippa, C. A., DeRuntz, J. A., 1977. Stabilization of staggered solution procedures for fluid-structure interaction analysis. Computational Methods for Fluid-Structure Interaction Problems 26, 95-124.

Patankar, S. V., Spalding, D. B., 1972. A calculation procedure for heat, mass and momentum transfer in three-dimensional parabolic flows. International Journal of Heat and Mass Transfer 15 (10), 1787-1806.

Piperno, S., Farhat, C., Larrouturou, B., 1995. Partitioned procedures for the transient solution of coupled aeroelastic problems Part I: Model problem, theory and two-dimensional application. Computer Methods in Applied Mechanics and Engineering 124, 79-112.

Rhie, C. M., Chow, W. L., 1983. Numerical study of the turbulent flow past an airfoil with trailing edge separation. AIAA Journal 21 (11), 1525-1532.

Smagorinsky, J., 1963. General circulation experiments with the primitive equations I: The basic experiment. Monthly Weather Review 91 (3), 99-165.

Spekreijse, S. P., 1995. Elliptic grid generation based on Laplace equations and algebraic transformations. Journal of Computational Physics 118 (1), 38-61.

Stone, H. L., 1968. Iterative solution of implicit approximations of multidimensional partial differential equations. SIAM Journal on Numerical Analysis 5 (3), 530-558.

Temam, R., 1969a. Sur l'approximation de la solution des équations de Navier-Stokes par la méthode des pas fractionnaires (i). Archive for Rational Mechanics and Analysis 32 (2), $135-153$.

Temam, R., 1969b. Sur l'approximation de la solution des équations de Navier-Stokes par la méthode des pas fractionnaires (ii). Archive for Rational Mechanics and Analysis 33 (5), $377-385$.

Thompson, J. F., Warsi, Z. U. A., Wayne Mastin, C. W., 1985. Numerical Grid Generation: Foundations and Applications. Vol. 45. North-Holland.

Turek, S., Hron, J., 2006. Proposal for numerical benchmarking of fluid-structure interaction between an elastic object and laminar incompressible flow. In: Bungartz, H.-J., Schäfer, M. (Eds.), Fluid-Structure Interaction. Vol. 53 of Lecture Notes in Computational Science and Engineering, LNCSE. Springer, Heidelberg, pp. 371-385.

Turek, S., Hron, J., Razzaq, M., Wobker, H., Schäfer, M., 2010. Numerical benchmarking of fluid-structure interaction: A comparison of different discretization and solution approaches. In: Bungartz, H.-J., Mehl, M., Schäfer, M. (Eds.), Fluid-Structure Interaction II - Modelling, Simulation, Optimization. Vol. 73 of Lecture Notes in Computational Science and Engineering, LNCSE. Springer, Heidelberg, pp. 413-424. 
Turek, S., Schäfer, M., 1996. Benchmark computations of laminar flow around a cylinder. In: Hirschel, E. H. (Ed.), Flow Simulation with High-Performance Computers II. Vol. 52 of Notes on Numerical Fluids Mechanics. Vieweg, Braunschweig, pp. 547-566.

Vierendeels, J., Degroote, J., Annerel, S., Haelterman, R., 2010. Stability issues in partitioned FSI calculations. In: Bungartz, H.-J., Mehl, M., Schäfer, M. (Eds.), Fluid-Structure Interaction II - Modelling, Simulation, Optimization. Vol. 73 of Lecture Notes in Computational Science and Engineering, LNCSE. Springer, Heidelberg, pp. 83-102.

Wüchner, R., 2006. Mechanik und Numerik der Formfindung und Fluid-Struktur-Interaktion von Membrantragwerken. Ph.D. thesis, Lehrstuhl für Statik, Technische Universität München, Germany.

Wüchner, R., Kupzok, A., Bletzinger, K.-U., 2007. A framework for stabilized partitioned analysis of thin membrane-wind interaction. International Journal for Numerical Methods in Fluids 54 (6-8), 945-963. 